\title{
Shifts in a phenanthrene-degrading microbial community are driven by carbohydrate metabolism selection in a ryegrass rhizosphere
}

Longfei Jiang, Chunling Luo ${ }^{\mathrm{a}, \mathrm{b} *}$, Dayi Zhang, Mengke Song ${ }^{\mathrm{b}}$, Weiping Mei $^{\mathrm{b}}$, Yingtao Sun a , Gan Zhang ${ }^{\mathrm{a}}$

a State Key Laboratory of Organic Geochemistry, Guangzhou Institute of Geochemistry, Chinese Academy of Sciences, Guangzhou 510640, China

b College of Natural Resources and Environment, South China Agricultural University, Guangzhou 510642, China

c School of Environment, Tsinghua University, Beijing 100084, China

* Corresponding author:

Prof. Chunling Luo, E-mail: clluo@gig.ac.cn; Tel.: +86-20-85290290; Fax: +86-20-85290706 


\section{Contents}

Table S1 The protein sequences downloaded from NCBI and UniProt used for protein annotation.

Table S2 Sugar concentration in ryegrass root exudates. .............................................15

Table S3 Organic acid concentration in ryegrass root exudates. .....................................16

Table S4 Taxonomic information of phenanthrene degraders.

Table S5 Relative abundance of all active phenanthrene degraders in NR and R treatments.

Table S6 The average $\mathrm{Ka} / \mathrm{Ks}$ ratios of phenanthrene degradation related genes and the $p$ values of one-way ANOVA

Table S7 The ten altered metabolic modules with top abundance and their corresponding $M$ value in Phenanthrene degraders and Total microbial community .20

Figure S1 The heights of all the cultivated plants.

Figure S2 The copy number of 16S rRNA genes (normalized to the highest abundance) along with $\mathrm{CsCl}$ gradient fractions in $\mathrm{NR}$ and $\mathrm{R}$ treatments.

Figure S3 Dominant microbes $(>1 \%$ ) on phyla level in R and NR treatments.

Figure S4 Dominant microbes $(>1 \%$ ) on genus level in R and NR treatments.

Figure S5 Score plot of PCoA for microbial communities from original soil, NR and R treatments.

Figure S6 Shannon index in original soil, NR and R treatments. .26

Figure S7 Residual phenanthrene percentage in R, NR and sterilized samples. .27

Figure S8 A) Phylogenetic tree of identified OTUs responsible for in situ phenanthrene degradation in NR and R treatments. B) Differences in phylogenetic distance between

NR and R treatments

Figure S9 The copy numbers of $16 \mathrm{~S}$ rRNA genes on day $0,3,7$ and 14 between R and NR treatments.

Figure S10 The 10 different abundance modules between NR and R treatments. .30

Figure S11 Root exudates metabolism related genes enriched in R treatment. 
Table S1 The protein sequences downloaded from NCBI and UniProt used for protein annotation.

\begin{tabular}{|c|c|}
\hline Gene & Sequence number \\
\hline $\begin{array}{l}\text { phenanthrene dioxygenase } \\
\text { gene }\end{array}$ & $\begin{array}{l}\text { WP_066762449.1, AOW72914.1, AOW72913.1, AGI05703.1, ACF95906.1, AGI05702.1, } \\
\text { WP_066762456.1 }\end{array}$ \\
\hline $\begin{array}{l}\text { phthalate-3,4-dihydrodiol } \\
\text { dehydrogenase gene }\end{array}$ & $\begin{array}{l}\text { UniRef100_A0A1B3EB20, UniRef100_A0A1H0Q8M6, UniRef100_A0A1H3LYK7, } \\
\text { UniRef100_A0A285E7T0, UniRef100_A1T2B3, UniRef100_E6TIQ4, } \\
\text { UniRef100_A0A1I1Q061, UniRef100_A0A1B3EAZ9, UniRef100_A0A0H5CWA9, } \\
\text { UniRef100_A0A2U0V6J5, UniRef100_A0A2N3ZRX6, UniRef100_A0A1M6XKE1, } \\
\text { UniRef100_A1UDE9, UniRef100_A0A1I4GNB2, UniRef100_A0A2T0ZYZ5, } \\
\text { UniRef100_A0A235G3V8, UniRef100_A0A1I5I4V1, UniRef100_A4T3E2 }\end{array}$ \\
\hline $\begin{array}{l}\text { phthalate-4,5-dioxygenase } \\
\text { gene }\end{array}$ & $\begin{array}{l}\text { UniRef100_A0A142MDY7, UniRef100_A0A1P8USQ5, UniRef100_A0A1Q2YMP2, } \\
\text { UniRef100_A0A1Y5TB63, UniRef100_A1VWM0, UniRef100_L2T4V5, } \\
\text { UniRef100_A0A160VBJ8, UniRef100_A0A1S9S056, UniRef100_A0A014MUG6, } \\
\text { UniRef100_A0A022MHH2, UniRef100_A0A024K5R3, UniRef100_A0A024QT10, } \\
\text { UniRef100_A0A0311935, UniRef100_A0A060PC92, UniRef100_A0A060PFY4, } \\
\text { UniRef100_A0A060PR29, UniRef100_A0A062V4S3, UniRef100_A0A063BA00, } \\
\text { UniRef100_A0A063BA49, UniRef100_A0A063BC93, UniRef100_A0A063BI08, } \\
\text { UniRef100_A0A063BPT0, UniRef100_A0A066UE62, UniRef100_A0A069I8R4, } \\
\text { UniRef100_A0A069NG40, UniRef100_A0A076MFB3, UniRef100_A0A076MNQ3, } \\
\text { UniRef100_A0A076MRJ0, UniRef100_A0A076MRK7, UniRef100_A0A076MVR2, } \\
\text { UniRef100_A0A076MZF3, UniRef100_A0A076MZW0, UniRef100_A0A076PRX6, } \\
\text { UniRef100_A0A081T110, UniRef100_A0A084SKT3, UniRef100_A0A094LUN9, } \\
\text { UniRef100_A0A094MB16, UniRef100_A0A098BIP9, UniRef100_A0A099KB31, } \\
\text { UniRef100_A0A099KGQ8, UniRef100_A0A099KUT8, UniRef100_A0A0A0N3N2, } \\
\text { UniRef100_A0A0A1Y7R8, UniRef100_A0A0A1YVP5, UniRef100_A0A0A6QJC7, } \\
\text { UniRef100_A0A0A6QJF7, UniRef100_A0A0A8BSG7, UniRef100_A0A0B3RY01, } \\
\text { UniRef100_A0A0B3S6L9, UniRef100_A0A0B5DWD5, UniRef100_A0A0B8MZK1, } \\
\text { UniRef100_A0A0B8NFT0, UniRef100_A0A0B9ASM1, UniRef100_A0A0C1Z2F1, } \\
\text { UniRef100_A0A0C2BYW3, UniRef100_A0A0C2C7H2, UniRef100_A0A0C2C863, } \\
\text { UniRef100_A0A0C4YMB1, UniRef100_A0A0C5JY70, UniRef100_A0A0D5LXG6, } \\
\text { UniRef100_A0A0E0ULX3, UniRef100_A0A0E3BRW0, UniRef100_A0A0E3U676, } \\
\text { UniRef100_A0A0E4H1G2, UniRef100_A0A0F0EX28, UniRef100_A0A0F0F3Z8, } \\
\text { UniRef100_A0A0F7E3I5, UniRef100_A0A0F7KZT4, UniRef100_A0A0G3ENU6, } \\
\text { UniRef100_A0A0H2M766, UniRef100_A0A0H2M9H7, UniRef100_A0A0H3WTC0, } \\
\text { UniRef100_A0A0H4W4R9, UniRef100_A0A0H4W9X5, UniRef100_A0A0I9YA52, } \\
\text { UniRef100_A0A019YX59, UniRef100_A0A0J1DD12, UniRef100_A0A0K2R881, } \\
\text { UniRef100_A0A0L0R218, UniRef100_A0A0L8LG47, UniRef100_A0A0L8Q3B1, } \\
\text { UniRef100_A0A0L8QLV1, UniRef100_A0A0M0EE72, UniRef100_A0A0M2L2C5, } \\
\text { UniRef100_A0A0M6YEY9, UniRef100_A0A0M8R900, UniRef100_A0A0M8RMB4, } \\
\text { UniRef100_A0A0N0MNT8, UniRef100_A0A0N0MZ99, UniRef100_A0A0N0NFA6, } \\
\text { UniRef100_A0A0N1G906, UniRef100_A0A0N1NBZ5, UniRef100_A0A0N1NRV2, }\end{array}$ \\
\hline
\end{tabular}


UniRef100_A0A0P0MB28, UniRef100_A0A0P0MCF4, UniRef100_A0A0P0MCI9, UniRef100_A0A0P0MI79, UniRef100_A0A0P0MIK3, UniRef100_A0A0P0MIW1, UniRef100_A0A0P0Z4H7, UniRef100_A0A0P1EH13, UniRef100_A0A0P1FN74, UniRef100_A0A0P1FRT5, UniRef100_A0A0P1GXM6, UniRef100_A0A0P1IKQ5, UniRef100_A0A0P9BE71, UniRef100_A0A0Q3I8S3, UniRef100_A0A0Q5R6K9, UniRef100_A0A0Q7HSL1, UniRef100_A0A0Q7KYW6, UniRef100_A0A0Q7TEB7, UniRef100_A0A0Q8QVI8, UniRef100_A0A0Q8X9W3, UniRef100_A0A0Q9MDH5, UniRef100_A0A0R3MWY7, UniRef100_A0A0S6UM99, UniRef100_A0A0S6WTD6, UniRef100_A0A0S9LXT7, UniRef100_A0A0S9MUX2, UniRef100_A0A0T1WIT2, UniRef100_A0A0T2YB98, UniRef100_A0A0T2YKG6, UniRef100_A0A0U0WAI4, UniRef100_A0A0U1CXN2, UniRef100_A0A0U2U4E3, UniRef100_A0A0U2VZX3, UniRef100_A0A0W7X7H1, UniRef100_A0A0X1T5I1, UniRef100_A0A0X3WD26, UniRef100_A0A0X3XNX1, UniRef100_A0A0X7JKF5, UniRef100_A0A100J671, UniRef100_A0A100W1W7, UniRef100_A0A101K3H2, UniRef100_A0A117EFD6, UniRef100_A0A127EN14, UniRef100_A0A127EPQ3, UniRef100_A0A142XJ29, UniRef100_A0A143QCZ0, UniRef100_A0A143QDY4, UniRef100_A0A146AMH9, UniRef100_A0A149PZ69, UniRef100_A0A154ML41, UniRef100_A0A160TK22, UniRef100_A0A160TX55, UniRef100_A0A179SJ78, UniRef100_A0A193BQG2, UniRef100_A0A193C6Y6, UniRef100_A0A193FS28, UniRef100_A0A193GM82, UniRef100_A0A193KXR5, UniRef100_A0A1A5XFM6, UniRef100_A0A1A9MY96, UniRef100_A0A1B1JWN3, UniRef100_A0A1B1K5E4, UniRef100_A0A1B1K877, UniRef100_A0A1B1KE41, UniRef100_A0A1B1KER3, UniRef100_A0A1B2GYV1, UniRef100_A0A1B2GYW2, UniRef100_A0A1B2R6W7, UniRef100_A0A1B5DDM1, UniRef100_A0A1B5DEL2, UniRef100_A0A1B5EAD8, UniRef100_A0A1B8NXQ4, UniRef100_A0A1B9VEH6, UniRef100_A0A1B9VEV9, UniRef100_A0A1C3GIW9, UniRef100_A0A1C3GT54, UniRef100_A0A1C3GU81, UniRef100_A0A1C3NYG6, UniRef100_A0A1D2UHI8, UniRef100_A0A1D7TW86, UniRef100_A0A1D8T017, UniRef100_A0A1D9H8W2, UniRef100_A0A1D9H935, UniRef100_A0A1E3EJU7, UniRef100_A0A1E3LQV6, UniRef100_A0A1E3ZJW7, UniRef100_A0A1E3ZK52, UniRef100_A0A1E4JZI9, UniRef100_A0A1E4PJ87, UniRef100_A0A1E4QM10, UniRef100_A0A1E7U3N5, UniRef100_A0A1E7WVS2, UniRef100_A0A1F4BEW5, UniRef100_A0A1F4EWY1, UniRef100_A0A1F4H6S8, UniRef100_A0A1F4J6R4, UniRef100_A0A1F4NEJ4, UniRef100_A0A1F4NFC7, UniRef100_A0A1F4NFP8, UniRef100_A0A1G4UF27, UniRef100_A0A1G4UF77, UniRef100_A0A1G4UFG3, UniRef100_A0A1G6LSS0, UniRef100_A0A1G6LT82, UniRef100_A0A1G9JRC0, UniRef100_A0A1G9JRI3, UniRef100_A0A1G9W4Z9, UniRef100_A0A1G9W532, UniRef100_A0A1H0GHS7, UniRef100_A0A1H0GHV5, UniRef100_A0A1H0Q817, UniRef100_A0A1H0Q8U5, UniRef100_A0A1H1IEQ8, UniRef100_A0A1H1IFW1, UniRef100_A0A1H1K920, UniRef100_A0A1H1K9U5, UniRef100_A0A1H1KHF3, UniRef100_A0A1H1KHS3, UniRef100_A0A1H4L5E1, UniRef100_A0A1H4MJZ8, UniRef100_A0A1H4RBK1, UniRef100_A0A1H4RBN8, UniRef100_A0A1H5ANP0, UniRef100_A0A1H5AQJ9, UniRef100_A0A1H6C3W4, UniRef100_A0A1H6C4V1, UniRef100_A0A1H6NW65, UniRef100_A0A1H9GTE6, UniRef100_A0A1H9J460, UniRef100_A0A1I0LB46, UniRef100_A0A1I2FB71, UniRef100_A0A1I2FD74, 
UniRef100_A0A1I4E4M3, UniRef100_A0A1I4E6I2, UniRef100_A0A1I5UYK2, UniRef100_A0A1I5UZB1, UniRef100_A0A1I7KBJ6, UniRef100_A0A1I7KBK2, UniRef100_A0A1J5P9Q0, UniRef100_A0A1K2FVH7, UniRef100_A0A1K2G2N8, UniRef100_A0A1L1PNU9, UniRef100_A0A1L6I3Y3, UniRef100_A0A1L6J8L0, UniRef100_A0A1L9NYF2, UniRef100_A0A1M2ZK54, UniRef100_A0A1M3DE23, UniRef100_A0A1M3JP14, UniRef100_A0A1M5NCZ6, UniRef100_A0A1M5ND67, UniRef100_A0A1M6HKI9, UniRef100_A0A1M6HKQ4, UniRef100_A0A1M6NCJ4, UniRef100_A0A1M7UET6, UniRef100_A0A1M7UEU3, UniRef100_A0A1M9H0E5, UniRef100_A0A1N3Y083, UniRef100_A0A1N7RNG2, UniRef100_A0A1N7SVS9, UniRef100_A0A1P8K4P4, UniRef100_A0A1P8USU7, UniRef100_A0A1P9Y054, UniRef100_A0A1P9YB53, UniRef100_A0A1Q2ZDI4, UniRef100_A0A1Q3HCW7, UniRef100_A0A1Q4BGK5, UniRef100_A0A1Q4BLK7, UniRef100_A0A1Q4CAF8, UniRef100_A0A1Q4E8S1, UniRef100_A0A1Q4VSS6, UniRef100_A0A1Q7BPZ5, UniRef100_A0A1Q7IJS0, UniRef100_A0A1Q8IWD9, UniRef100_A0A1Q8J0I5, UniRef100_A0A1Q9C206, UniRef100_A0A1Q9LJK6, UniRef100_A0A1Q9R6S2, UniRef100_A0A1Q9R8B3, UniRef100_A0A1R0KVN1, UniRef100_A0A1R0L144, UniRef100_A0A1R1JZI0, UniRef100_A0A1S2SBH0, UniRef100_A0A1S8FSN2, UniRef100_A0A1S8FTA2, UniRef100_A0A1S8FTG8, UniRef100_A0A1S9E1L7, UniRef100_A0A1T4Y5Z5, UniRef100_A0A1U2Z656,UniRef100_A0A1U9Z9L8, UniRef100_A0A1V1VZ86, UniRef100_A0A1V1W9P9, UniRef100_A0A1V2Q004, UniRef100_A0A1V4AAI9, UniRef100_A0A1V4D7T6, UniRef100_A0A1V6FE45, UniRef100_A0A1W2DPD9, UniRef100_A0A1W2LGY2, UniRef100_A0A1W2M3M6, UniRef100_A0A1W6ZPF3, UniRef100_A0A1W7A133, UniRef100_A0A1W7M461, UniRef100_A0A1W7M6X8, UniRef100_A0A1W7MBP9, UniRef100_A0A1X2DMY0, UniRef100_A0A1X2FHI8, UniRef100_A0A1X6Y3A0, UniRef100_A0A1X6Y3B6, UniRef100_A0A1X6YZ16, UniRef100_A0A1X6Z162, UniRef100_A0A1X6ZC43, UniRef100_A0A1X6ZXM4, UniRef100_A0A1X7A757, UniRef100_A0A1X7ADG3, UniRef100_A0A1X7KW71, UniRef100_A0A1X9UDA1, UniRef100_A0A1Y0T2Q4, UniRef100_A0A1Y2N774, UniRef100_A0A1Y5PTI0, UniRef100_A0A1Y5TX01, UniRef100_A0A1Y5TXB5, UniRef100_A0A1Y6CMZ9, UniRef100_A0A1Y6CUF5, UniRef100_A0A1Y6FHD6, UniRef100_A0A1Y6FQF1, UniRef100_A0A1Y6KWD0, UniRef100_A0A1Y6MD88, UniRef100_A0A1Y6MF41, UniRef100_A0A1Z1W3J7, UniRef100_A0A1Z9DB35, UniRef100_A0A208XGG0, UniRef100_A0A208XKT8, UniRef100_A0A208XKW0, UniRef100_A0A208XQF2, UniRef100_A0A208XQH1, UniRef100_A0A208XZ82, UniRef100_A0A222EA73, UniRef100_A0A222T909, UniRef100_A0A225M3K2, UniRef100_A0A226WZX0, UniRef100_A0A229RWT3, UniRef100_A0A229SH12, UniRef100_A0A235FHR0, UniRef100_A0A235FIF0, UniRef100_A0A238D146, UniRef100_A0A238JK32, UniRef100_A0A238KIJ7, UniRef100_A0A238KIL9, UniRef100_A0A239F0J0, UniRef100_A0A239F0R3, UniRef100_A0A240U027, UniRef100_A0A243VFV7, UniRef100_A0A244DL42, UniRef100_A0A250DHG3, UniRef100_A0A250IE52, UniRef100_A0A258GES6, UniRef100_A0A261RQX6, UniRef100_A0A286NVQ5, UniRef100_A0A290ZQA2, UniRef100_A0A291RF65, UniRef100_A0A291SSM9, UniRef100_A0A291T3Q0, UniRef100_A0A292YM70, UniRef100_A0A292YYQ9, UniRef100_A0A292Z463, 
UniRef100_A0A2A1ZUI5, UniRef100_A0A2A2A2P1, UniRef100_A0A2A2V6W3, UniRef100_A0A2B8BN36, UniRef100_A0A2C9BM14, UniRef100_A0A2C9BNJ1, UniRef100_A0A2C9BNX0, UniRef100_A0A2C9BXE3, UniRef100_A0A2C9DDG1, UniRef100_A0A2C9ZSQ4, UniRef100_A0A2D6G5I0, UniRef100_A0A2D6I238, UniRef100_A0A2D8B624, UniRef100_A0A2D8B7X7, UniRef100_A0A2E2K8K8, UniRef100_A0A2E2ZRS4, UniRef100_A0A2E4EYT6, UniRef100_A0A2E4EZP2, UniRef100_A0A2E4F138, UniRef100_A0A2E4F166, UniRef100_A0A2E6UFE6, UniRef100_A0A2E6UIC8, UniRef100_A0A2E7C3A6, UniRef100_A0A2E7QIA9, UniRef100_A0A2E8PC42, UniRef100_A0A2E8UZ33, UniRef100_A0A2E9BPE2, UniRef100_A0A2J7VS12, UniRef100_A0A2J7YJ52, UniRef100_A0A2M6VFC4, UniRef100_A0A2M6VMQ9, UniRef100_A0A2M6VY89, UniRef100_A0A2N7W2A6, UniRef100_A0A2N7Y8H7, UniRef100_A0A2N7ZDW1, UniRef100_A0A2N8MPW4, UniRef100_A0A2N8MUY8, UniRef100_A0A2P2C8M5, UniRef100_A0A2P2FRK0, UniRef100_A0A2P2FSV8, UniRef100_A0A2P7ZZQ7, UniRef100_A0A2R8B9I3, UniRef100_A0A2R8C994, UniRef100_A0A2S0MFG0, UniRef100_A0A2S0XSM2, UniRef100_A0A2S4KB87, UniRef100_A0A2S6PUY9, UniRef100_A0A2S6Q7T3, UniRef100_A0A2S6Q9V8, UniRef100_A0A2S6QA16, UniRef100_A0A2S6TEL0, UniRef100_A0A2S6TEN9, UniRef100_A0A2S6TGK7, UniRef100_A0A2S6TMQ5, UniRef100_A0A2S6TXZ2, UniRef100_A0A2S6XEN6, UniRef100_A0A2S9K4J8, UniRef100_A0A2S9KAS2, UniRef100_A0A2T0KT18, UniRef100_A0A2T0MSV0, UniRef100_A0A2T0R3Y0, UniRef100_A0A2T0VBK4, UniRef100_A0A2T3JVU7, UniRef100_A0A2T3K7Z6, UniRef100_A0A2T3PSH3, UniRef100_A0A2T6F9K3, UniRef100_A0A2U1CLM3, UniRef100_A0A2U1CP33, UniRef100_A0A2U3MVQ6, UniRef100_A0A2U3N3C8, UniRef100_A0JVQ0, UniRef100_A1VWL7, UniRef100_A1WM04, UniRef100_A1WM07, UniRef100_A1WNU5, UniRef100_A2SP36, UniRef100_A5VAU6, UniRef100_A5VEC1, UniRef100_A7IE52, UniRef100_A8M132, UniRef100_A9BSJ5, UniRef100_B1FCG2, UniRef100_B1K673, UniRef100_B1T279, UniRef100_B1Y697, UniRef100_B2JRN4, UniRef100_B2JSG7, UniRef100_B2JTT5, UniRef100_B3QGW7, UniRef100_B5WFJ8, UniRef100_B5WFK0, UniRef100_B5WJ27, UniRef100_B5WQ71, UniRef100_B5WVR1, UniRef100_B7X5F0, UniRef100_B9NY84, UniRef100_B9TM69, UniRef100_C4RH27, UniRef100_C5CZN4, UniRef100_D5WF29, UniRef100_D5WGK6, UniRef100_D9V9K2, UniRef100_F2JU25, UniRef100_F2JV69, UniRef100_F4CJK8, UniRef100_F4CN75, UniRef100_F4CP94, UniRef100_F4CPQ7, UniRef100_F4CYA0, UniRef100_F4F993, UniRef100_F5NUF8, UniRef100_F5XWD6, UniRef100_F6AJ53, UniRef100_F6AP67, UniRef100_F6AP70, UniRef100_F6AP73, UniRef100_F6APX6, UniRef100_F6AWH0, UniRef100_F6CY07, UniRef100_F6EWC9, UniRef100_F8AWK8, UniRef100_F8AZZ5, UniRef100_F8B5U5, UniRef100_F8GSL4, UniRef100_G0FMD2, UniRef100_G0FSX0, UniRef100_G0FUL2, UniRef100_G0FUL3, UniRef100_G0FZR9, UniRef100_G0PWX2, UniRef100_G2IPJ1, UniRef100_G2IT77, UniRef100_G4HQC1, UniRef100_G4HS51, UniRef100_G4HWJ9, UniRef100_G4I7Q0, UniRef100_G4I8M7, UniRef100_G7CJ83, UniRef100_G8MJ19, UniRef100_G8MLU7, UniRef100_H0FW62, UniRef100_H0G516, UniRef100_H1S1C2, UniRef100_H1S7I0, UniRef100_H1SEV3, UniRef100_H6RMM9, UniRef100_H6RX64, UniRef100_H8IQ36, UniRef100_I0RXH6, UniRef100_I0W5Y7, UniRef100_I0W6D5, UniRef100_I0W7Z9, UniRef100_I0WN15, 
UniRef100_I0WPP6, UniRef100_I2JIC7, UniRef100_I4EXN9, UniRef100_I4EXP4, UniRef100_I5CXI0, UniRef100_I7FHX6, UniRef100_J0JDJ8, UniRef100_J0W7T5, UniRef100_J4TIH6, UniRef100_K0DNR2, UniRef100_K0EWT0, UniRef100_K0I0T4, UniRef100_K0V4V5, UniRef100_K2N0U8, UniRef100_K6X401, UniRef100_K8X633, UniRef100_K8X950, UniRef100_K8XAK1, UniRef100_K8XJ60, UniRef100_K8XLS5, UniRef100_K8XM39, UniRef100_K8XP82, UniRef100_K8XV50, UniRef100_K8Y0Y5, UniRef100_K9ART6, UniRef100_L2T626, UniRef100_L2T9P7, UniRef100_L2TF57, UniRef100_L2TM31, UniRef100_L2TNK1, UniRef100_L2TQG1, UniRef100_L2TRQ6, UniRef100_L2TSX2, UniRef100_L2TVK5, UniRef100_L7T1F7, UniRef100_L7T545, UniRef100_L7T549, UniRef100_L8EZB0, UniRef100_L8P5A4, UniRef100_M2X6Y1, UniRef100_M2YQQ0, UniRef100_M2YR49, UniRef100_M2ZJX9, UniRef100_M4X3V7, UniRef100_N9WH91, UniRef100_Q05182, UniRef100_Q05183, UniRef100_Q0S9K5, UniRef100_Q0SJI0, UniRef100_Q12C99, UniRef100_Q12CA0, UniRef100_Q143N4, UniRef100_Q143N9, UniRef100_Q1LBR9, UniRef100_Q46MK3, UniRef100_Q46P10, UniRef100_Q46P14, UniRef100_Q46RU1, UniRef100_R1G455, UniRef100_R1GFM5, UniRef100_R4T0C5, UniRef100_R4TJV9, UniRef100_R4X4G8, UniRef100_R7WJ29, UniRef100_R7WK50, UniRef100_S4ZBG4, UniRef100_S5YCA7, UniRef100_U3QQ57, UniRef100_U3R2L0, UniRef100_V4YH77, UniRef100_W1S2L5, UniRef100_W3ZX40, UniRef100_W3ZZB0, UniRef100_W4A323, UniRef100_W4A3K0, UniRef100_W4A5A6, UniRef100_W4A8A4, UniRef100_W4AB21, UniRef100_W4ADG9, UniRef100_W4LJ52, UniRef100_W6WEF5, UniRef100_W7SNJ9, UniRef100_W7TD49, UniRef100_W7W797, UniRef100_W7W834, UniRef100_W7WBL8, UniRef100_W7WKZ8, UniRef100_W7X255, UniRef100_W8HGN1, UniRef100_W9B2B1

salicylate hydroxylase UniRef100_UPI00083B8E79, UniRef100_UPI00083AB5BE, UniRef100_UPI000837641B, UniRef100_UPI00083DD161, UniRef100_UPI00083B2BF9, UniRef100_UPI0008414F93, UniRef100_UPI0008417610, UniRef100_UPI000841E720, UniRef100_UPI00082B69B2, UniRef100_UPI00082BD5BE, UniRef100_UPI000824BBFC, UniRef100_UPI00082B65AB, UniRef100_UPI00082BAFC3, UniRef100_UPI0007864C91, UniRef100_UPI0007B5420F, UniRef100_UPI0008303D6B, UniRef100_UPI0008325C15, UniRef100_UPI0007813F84, UniRef100_UPI0007848486, UniRef100_UPI00078090CD, UniRef100_UPI000782AFA7, UniRef100_UPI0008304198, UniRef100_UPI00082E4D71, UniRef100_UPI0008254EFD, UniRef100_UPI0008244DA1, UniRef100_UPI000824A8BE, UniRef100_A0A017S3U6, UniRef100_A0A017S871, UniRef100_A0A017SEJ7, UniRef100_A0A023XV13, UniRef100_A0A023XVD3, UniRef100_A0A024LUP8, UniRef100_A0A024M897, UniRef100_A0A024M8A7, UniRef100_A0A024M8C6, UniRef100_A0A024QRY0, UniRef100_A0A063C154, UniRef100_A0A066XG96, UniRef100_A0A066XI90, UniRef100_A0A066XXU8, UniRef100_A0A074X7V1, UniRef100_A0A074X9I9, UniRef100_A0A084GE57, UniRef100_A0A090CI48, UniRef100_A0A0G2DW97, UniRef100_A0A0G2ETG1, UniRef100_A0A0G2EX91, UniRef100_A0A0G2F8H2, UniRef100_A0A0G2FDV1, UniRef100_A0A0G2FLV0, UniRef100_A0A0G2FMU6, UniRef100_A0A0G2FPC9, UniRef100_A0A0G2FSV8, UniRef100_A0A0G2FU68, UniRef100_A0A0G2FWA4, UniRef100_A0A0G2G055, UniRef100_A0A0G2GWE1, UniRef100_A0A0G2HXJ8, UniRef100_A0A0G2I8M9, UniRef100_A0A0H5RIW7, UniRef100_A0A0H5RKQ9, UniRef100_A0A0J5PKZ1, UniRef100_A0A0K3CU17, 
UniRef100_A0A0L1IL01, UniRef100_A0A0L1IS42, UniRef100_A0A0L1ITG6, UniRef100_A0A0L1IUC7, UniRef100_A0A0S4X2M4, UniRef100_A0A0T9MXK7, UniRef100_A0A0U0YCB2, UniRef100_A0A0U1AU70, UniRef100_A0A0U1AVU0, UniRef100_A0A0U1DP97, UniRef100_A0A0U1DTE1, UniRef100_A0A0U3AQI7, UniRef100_A0A0W0FUL0, UniRef100_A0A100W4K8, UniRef100_A0A136IPX7, UniRef100_A0A136IYS3, UniRef100_A0A136JA56, UniRef100_A0A142I725, UniRef100_A0A146FYN4, UniRef100_A0A177CH14, UniRef100_A0A177CP71, UniRef100_A0A177DZJ4, UniRef100_A0A178AXU7, UniRef100_A0A178ERL1, UniRef100_A0A178FKZ4, UniRef100_A0A179FSU7, UniRef100_A0A179H7X2, UniRef100_A0A179U4P9, UniRef100_A0A179UTV6, UniRef100_A0A194WVC6, UniRef100_A0A1A7MJ70, UniRef100_A0A1A7MMD3, UniRef100_A0A1B7YLD3, UniRef100_A0A1C1C7F1, UniRef100_A0A1C1C8K0, UniRef100_A0A1C1CC33, UniRef100_A0A1C1CE64, UniRef100_A0A1C1CGP1, UniRef100_A0A1C1CMW0, UniRef100_A0A1C1CN04, UniRef100_A0A1C1CXJ6, UniRef100_A0A1E1KI30, UniRef100_A0A1E1KL49, UniRef100_A0A1E1LBH3, UniRef100_A0A1E1LCQ7, UniRef100_A0A1E1LD25, UniRef100_A0A1E1LJS0, UniRef100_A0A1E1LUN2, UniRef100_A0A1E1LWG4, UniRef100_A0A1E1MU65, UniRef100_A0A1F7ZT45, UniRef100_A0A1F7ZUY6, UniRef100_A0A1F8AB74, UniRef100_A0A1L7SH63, UniRef100_A0A1L7T1V6, UniRef100_A0A1L7T5I3, UniRef100_A0A1L7T8N6, UniRef100_A0A1L7TNJ2, UniRef100_A0A1L7TQF4, UniRef100_A0A1L7U7G8, UniRef100_A0A1L7UAE2, UniRef100_A0A1L7UCQ2, UniRef100_A0A1L7UJ08, UniRef100_A0A1L7UMQ4, UniRef100_A0A1L7UYR3, UniRef100_A0A1L7V944, UniRef100_A0A1L7VJI4, UniRef100_A0A1L7VRL2, UniRef100_A0A1L7VTH4, UniRef100_A0A1L7VTV6, UniRef100_A0A1L7VU06, UniRef100_A0A1L7VYU0, UniRef100_A0A1L7VZ20, UniRef100_A0A1L7W5W1, UniRef100_A0A1L7W6D4, UniRef100_A0A1L7W8Y4, UniRef100_A0A1L7WBZ1, UniRef100_A0A1L7WF16, UniRef100_A0A1L7WLF8, UniRef100_A0A1L7WVF1, UniRef100_A0A1L7XA82, UniRef100_A0A1L7XCX6, UniRef100_A0A1L7XEZ7, UniRef100_A0A1L7XIB9, UniRef100_A0A1L7XJ89, UniRef100_A0A1L7XLL2, UniRef100_A0A1L7XPK1, UniRef100_A0A1L7XPP8, UniRef100_A0A1L7XQV7, UniRef100_A0A1L7XRD9, UniRef100_A0A1L7XRG1, UniRef100_A0A1L7XSI5, UniRef100_A0A1L7XVG0, UniRef100_A0A1M8GK26, UniRef100_A0A1M8X3Y8, UniRef100_A0A1M9B8K5, UniRef100_A0A1M9E826, UniRef100_A0A1S7UJK2, UniRef100_A0A1S7UMN9, UniRef100_A0A1S8A554, UniRef100_A0A1S8A887, UniRef100_A0A1S8A8H4, UniRef100_A0A1S8ABJ7, UniRef100_A0A1S9RTD8, UniRef100_A0A1S9RV99, UniRef100_A0A1S9RVD2, UniRef100_A0A1S9RZK9, UniRef100_A0A1S9S278, UniRef100_A0A1T3CD03, UniRef100_A0A1T9G319, UniRef100_A0A1W2TF28, UniRef100_A0A1W2TF53, UniRef100_A0A1W2THA0, UniRef100_A0A1W2TVE9, UniRef100_A0A1Y1Z350, UniRef100_A0A1Y2DCH9, UniRef100_A0A1Y2DJ02, UniRef100_A0A1Y2DW97, UniRef100_A0A1Y2T850, UniRef100_A0A1Z4F064, UniRef100_A0A284RKI7, UniRef100_A0A2D3ULU4, UniRef100_A0A2D3UP79, UniRef100_A0A2D3UV85, UniRef100_A0A2D3UYM6, UniRef100_A0A2D3VEW4, UniRef100_A0A2D3VJ83, UniRef100_A0A2H2ZDE8, UniRef100_A0A2H3RD33, UniRef100_A0A2H3RKR5, UniRef100_A0A2H3RUL3, UniRef100_A0A2H3RUV1, 
UniRef100_A0A2H3S3I5, UniRef100_A0A2H3S7P3, UniRef100_A0A2H3S7U5, UniRef100_A0A2H3S9K9, UniRef100_A0A2H3SFI1, UniRef100_A0A2H3SI32, UniRef100_A0A2H3SPA2, UniRef100_A0A2H3SRX6, UniRef100_A0A2H3SU27, UniRef100_A0A2H3SYC1, UniRef100_A0A2H3T294, UniRef100_A0A2H3T2H9, UniRef100_A0A2H3T7P6, UniRef100_A0A2H3TRF6, UniRef100_A0A2H3TSV3, UniRef100_A0A2H3TW53, UniRef100_A0A2H3TW72, UniRef100_A0A2H3U0Q4, UniRef100_A0A2I0RTF4, UniRef100_A0A2I1BSV4, UniRef100_A0A2I1BVF4, UniRef100_A0A2I1BVF9, UniRef100_A0A2I1BWF2, UniRef100_A0A2I1C438, UniRef100_A0A2I1C910, UniRef100_A0A2I1CI43, UniRef100_A0A2I1CL57, UniRef100_A0A2I1CLF5, UniRef100_A0A2I2FRY0, UniRef100_A0A2I2FUI0, UniRef100_A0A2I2FZU1, UniRef100_A0A2I2G2V4, UniRef100_A0A2I2G3H5, UniRef100_A0A2I2G773, UniRef100_A0A2I2GKD1, UniRef100_A0A2J6QE58, UniRef100_A0A2J6TM97, UniRef100_A0A2P2GX78, UniRef100_A0A2P2HC41, UniRef100_A0A2P2HEA3, UniRef100_A0A2P2HFG4, UniRef100_A0A2P2HLQ0, UniRef100_A0A2P2HNH9, UniRef100_A0A2T3A633, UniRef100_A0A2T3AH53, UniRef100_A0A2U3E9I6, UniRef100_A8IEW2, UniRef100_A8LNB4, UniRef100_A9I1A6, UniRef100_A9IEJ6, UniRef100_B0Y4Y9, UniRef100_B3PYM5, UniRef100_B6QD12, UniRef100_B8MC40, UniRef100_B8NQS2, UniRef100_C7CK85, UniRef100_C8VQ56, UniRef100_C9Z9P9, UniRef100_D4ALB3, UniRef100_D4DKY5, UniRef100_D8N0T2, UniRef100_D8NEP7, UniRef100_E4ZUW6, UniRef100_E5A0T8, UniRef100_E5A375, UniRef100_E5A5Q6, UniRef100_E5A5T4, UniRef100_E9ECJ3, UniRef100_F2QXP1, UniRef100_F2QXP4, UniRef100_F2T5Y3, UniRef100_F3ZBY3, UniRef100_G2XZR7, UniRef100_G2Y004, UniRef100_G2YMB3, UniRef100_G2YVA1, UniRef100_G7XVK0, UniRef100_H1VLM5, UniRef100_H2K4J9, UniRef100_I4EXP3, UniRef100_J3KDG5, UniRef100_K0PGC8, UniRef100_L8F3E8, UniRef100_M1VVU7, UniRef100_M7SEP0, UniRef100_M7SER7, UniRef100_M7SJ30, UniRef100_M7SK09, UniRef100_M7SNZ1, UniRef100_M7STP4, UniRef100_M7SY86, UniRef100_M7SZE2, UniRef100_M7SZI1, UniRef100_M7T1Y6, UniRef100_M7T9G7, UniRef100_M7TBL3, UniRef100_M7TCZ8, UniRef100_M7TGI9, UniRef100_M7TGX2, UniRef100_M7TH68, UniRef100_M7TP42, UniRef100_M7TQV0, UniRef100_M7U8S0, UniRef100_M7UBX7, UniRef100_M7UJ60, UniRef100_M7UNH0, UniRef100_M7UT08,UniRef100_M7UTY5, UniRef100_M7V3W3, UniRef100_Q1MH69, UniRef100_Q2CHW1, UniRef100_Q2L206, UniRef100_Q4WQS6, UniRef100_Q8FUA4, UniRef100_Q9Z4Y6, UniRef100_R1EBZ4, UniRef100_R1ELB9, UniRef100_R1FW60, UniRef100_R1G024, UniRef100_R1GDK1, UniRef100_R1GHC9, UniRef100_R1GJ48, UniRef100_R8BDU1, UniRef100_R8BDU2, UniRef100_R8BGW5, UniRef100_R8BIJ6, UniRef100_R8BNX0, UniRef100_R8BT76, UniRef100_R8BU70, UniRef100_R8BVH1, UniRef100_R8BWU8, UniRef100_S0DVT1, UniRef100_S0E365, UniRef100_S0EHM6, UniRef100_S0EKI6, UniRef100_S0EL80, UniRef100_S0EPN9, UniRef100_S0EQ05, UniRef100_T5AP91, UniRef100_U2XP03, UniRef100_UPI0001A9F698, UniRef100_UPI0001A9FCE7, UniRef100_UPI0001B558F8, UniRef100_UPI0001F2660C, UniRef100_UPI0001F26AA6, UniRef100_UPI0001F26AAC, UniRef100_UPI0001F2766F, UniRef100_UPI0002726D40, UniRef100_UPI0002887199, UniRef100_UPI0002A31E98, UniRef100_UPI0002D6ECA4, UniRef100_UPI0002EBF1AE, UniRef100_UPI0002F34CC7, UniRef100_UPI0002F687D9, UniRef100_UPI00030377C6, UniRef100_UPI0003040178, 
UniRef100_UPI0003046EE1, UniRef100_UPI000319AD73, UniRef100_UPI00031B193F, UniRef100_UPI000349C913, UniRef100_UPI00034C4E0A, UniRef100_UPI00035DEBA6, UniRef100_UPI000365D933, UniRef100_UPI000366EAF2, UniRef100_UPI0003677E83, UniRef100_UPI0003678B9F, UniRef100_UPI000369A5CD, UniRef100_UPI00036AD69D, UniRef100_UPI00036E1B9D, UniRef100_UPI00036F120C, UniRef100_UPI00037286D6, UniRef100_UPI00037AA0D0, UniRef100_UPI00037B56FE, UniRef100_UPI00037C5926, UniRef100_UPI00037E4535, UniRef100_UPI000380320F, UniRef100_UPI0003A15F62, UniRef100_UPI0003A9B19E, UniRef100_UPI0003AA52C8, UniRef100_UPI0003B633F6, UniRef100_UPI0003CE1A38, UniRef100_UPI0003CE2217, UniRef100_UPI0003D23349, UniRef100_UPI0003E2EC60, UniRef100_UPI0003F571D5, UniRef100_UPI0003FC0A6D, UniRef100_UPI0003FC6E74, UniRef100_UPI0003FE2E21, UniRef100_UPI0003FE92EE, UniRef100_UPI0004001169, UniRef100_UPI0004052A47, UniRef100_UPI000408C7D0, UniRef100_UPI00040B158E, UniRef100_UPI00040D800E, UniRef100_UPI00040ECA30, UniRef100_UPI00041588E6, UniRef100_UPI0004190D22, UniRef100_UPI00041BEE86, UniRef100_UPI00041E7EA0, UniRef100_UPI00041E859C, UniRef100_UPI00041FB288, UniRef100_UPI000421FB4C, UniRef100_UPI000422CEA3, UniRef100_UPI0004232E07, UniRef100_UPI0004244C72, UniRef100_UPI000424790E, UniRef100_UPI000425060D, UniRef100_UPI000425546F, UniRef100_UPI0004278F66, UniRef100_UPI000429BE3C, UniRef100_UPI00042A5041, UniRef100_UPI00043763AB, UniRef100_UPI0004409D7B, UniRef100_UPI000440D8EF, UniRef100_UPI0004412C46, UniRef100_UPI00044B4942, UniRef100_UPI00044EB1A6, UniRef100_UPI000455D6F4, UniRef100_UPI000455DA32, UniRef100_UPI000455FEA2, UniRef100_UPI0004624746, UniRef100_UPI000462FB3D, UniRef100_UPI0004691233, UniRef100_UPI00046936F7, UniRef100_UPI000469A959, UniRef100_UPI00046A32CD, UniRef100_UPI00046AC50D, UniRef100_UPI0004777BEE, UniRef100_UPI0004796CA6, UniRef100_UPI000479D03D, UniRef100_UPI00047B9EB0, UniRef100_UPI00047D3335, UniRef100_UPI00047F372D, UniRef100_UPI0004812E22, UniRef100_UPI0004823638, UniRef100_UPI00048279D6, UniRef100_UPI000483D32E, UniRef100_UPI00048444C8, UniRef100_UPI000485739F, UniRef100_UPI00048598F2, UniRef100_UPI0004860542, UniRef100_UPI000487FE04, UniRef100_UPI00048ABE12, UniRef100_UPI00048ACF74, UniRef100_UPI00048AEC4D, UniRef100_UPI00048E3156, UniRef100_UPI000491CFAD, UniRef100_UPI000493D773, UniRef100_UPI000494D4B4, UniRef100_UPI0004955942, UniRef100_UPI00049763CF, UniRef100_UPI0004A9A288, UniRef100_UPI0004AAF0BE, UniRef100_UPI0004AB8B24, UniRef100_UPI0004B5AAB8, UniRef100_UPI0004B791CA, UniRef100_UPI0004BB89A6, UniRef100_UPI0004C3F9AF, UniRef100_UPI0004C475BD, UniRef100_UPI0004C4D596, UniRef100_UPI0004C66421, UniRef100_UPI0004C97874, UniRef100_UPI0004CA227E, UniRef100_UPI0004CF6899, UniRef100_UPI0004D72E83, UniRef100_UPI0004F758F9, UniRef100_UPI000500CB49, UniRef100_UPI00050E4CE2, UniRef100_UPI00050F6863, UniRef100_UPI0005190779, UniRef100_UPI00051C3075, UniRef100_UPI000521162E, UniRef100_UPI000522F708, UniRef100_UPI000539110D, UniRef100_UPI00054AD857, UniRef100_UPI00054D26F5, UniRef100_UPI00054DE8C0, UniRef100_UPI00054F18F3, UniRef100_UPI00054F75E2, UniRef100_UPI0005525500, UniRef100_UPI000552BAC0, UniRef100_UPI0005535C55, UniRef100_UPI00055404BE, UniRef100_UPI0005548910, UniRef100_UPI00055A0BE4, UniRef100_UPI00055A2A82, UniRef100_UPI00055A4F09, UniRef100_UPI00055D44DF, 
UniRef100_UPI00056187BE, UniRef100_UPI00056246B3, UniRef100_UPI0005637B21, UniRef100_UPI000565BC6D, UniRef100_UPI000566B30B, UniRef100_UPI00056A5A50, UniRef100_UPI00056B620E, UniRef100_UPI00056BCCE2, UniRef100_UPI00056C1466, UniRef100_UPI00056CA76E, UniRef100_UPI00056E529F, UniRef100_UPI00056F494A, UniRef100_UPI00056F7A22, UniRef100_UPI00056F9528, UniRef100_UPI00057151FA, UniRef100_UPI0005715AF4, UniRef100_UPI0005793E51, UniRef100_UPI0005798E9F, UniRef100_UPI000579E17E, UniRef100_UPI00057EE853, UniRef100_UPI00058B23DB, UniRef100_UPI00059566B9, UniRef100_UPI00059C6261, UniRef100_UPI0005ABC025, UniRef100_UPI0005ACFA0B, UniRef100_UPI0005B39D7D, UniRef100_UPI0005B43BF4, UniRef100_UPI0005B54D03, UniRef100_UPI0005B81AB8, UniRef100_UPI0005BA6C29, UniRef100_UPI0005BA8E13, UniRef100_UPI0005BC4394, UniRef100_UPI0005BDB5D8, UniRef100_UPI0005C1C11A, UniRef100_UPI0005C1CEBC, UniRef100_UPI0005D7EDF8, UniRef100_UPI0005D7FC7E, UniRef100_UPI0005D990BA, UniRef100_UPI0005F8108D, UniRef100_UPI0005F99BD1, UniRef100_UPI0006210F98, UniRef100_UPI0006211EC1, UniRef100_UPI0006216F07, UniRef100_UPI00062A1286, UniRef100_UPI00062A3D8D, UniRef100_UPI00063CE689, UniRef100_UPI0006403D94, UniRef100_UPI000641B29D, UniRef100_UPI0006455731, UniRef100_UPI000645C948, UniRef100_UPI000645E780, UniRef100_UPI000646C403, UniRef100_UPI000646F39F, UniRef100_UPI0006474544, UniRef100_UPI000647DC9F, UniRef100_UPI000648B9E1, UniRef100_UPI000648D056, UniRef100_UPI0006528B42, UniRef100_UPI0006528FBA, UniRef100_UPI00065CAB60, UniRef100_UPI00065E2102, UniRef100_UPI00065E4CC0, UniRef100_UPI0006681826, UniRef100_UPI0006732FD1, UniRef100_UPI00067CD1B8, UniRef100_UPI00067CFCAF, UniRef100_UPI00067D166B, UniRef100_UPI00067FF9AE, UniRef100_UPI00068598A4, UniRef100_UPI000685F676, UniRef100_UPI00068C4655, UniRef100_UPI00068C6B44, UniRef100_UPI00068DC2C0, UniRef100_UPI00068F106F, UniRef100_UPI00068F82F2, UniRef100_UPI00068FEEB2, UniRef100_UPI000691B564, UniRef100_UPI0006AC1144, UniRef100_UPI0006B32CFD, UniRef100_UPI0006B3421C, UniRef100_UPI0006BEE735, UniRef100_UPI0006BF2914, UniRef100_UPI0006BFE8D9, UniRef100_UPI0006C3E057, UniRef100_UPI0006C639A9, UniRef100_UPI0006C7730D, UniRef100_UPI0006C83D55, UniRef100_UPI0006C8474C, UniRef100_UPI0006C87D5B, UniRef100_UPI0006C8944B, UniRef100_UPI0006C89F13, UniRef100_UPI0006C8BF5A, UniRef100_UPI0006C8D9BE, UniRef100_UPI0006DCF73F, UniRef100_UPI0006DD0C48, UniRef100_UPI0006E29B64, UniRef100_UPI0006FD35F5, UniRef100_UPI0006FE660E, UniRef100_UPI00070B4967, UniRef100_UPI00070C237D, UniRef100_UPI00070C30DD, UniRef100_UPI00070CFF09, UniRef100_UPI00070DE748, UniRef100_UPI000713A76E, UniRef100_UPI0007153B3F, UniRef100_UPI000715E3EB, UniRef100_UPI00071874B9, UniRef100_UPI000721761F, UniRef100_UPI0007350C11, UniRef100_UPI000735370C, UniRef100_UPI000735A2D5, UniRef100_UPI00073D7BF1, UniRef100_UPI000742F841, UniRef100_UPI000752CD5E, UniRef100_UPI000753FD93, UniRef100_UPI00075457A3, UniRef100_UPI000755CD3C, UniRef100_UPI0007562AAB, UniRef100_UPI000756D139, UniRef100_UPI000757ACA1, UniRef100_UPI000758454C, UniRef100_UPI0007587D86, UniRef100_UPI000758EAFF, UniRef100_UPI000759269D, UniRef100_UPI000759666F, UniRef100_UPI000759D110, UniRef100_UPI00075A014A, UniRef100_UPI00075A2722, UniRef100_UPI00075A43EB, UniRef100_UPI00075B0941, UniRef100_UPI00075B3CFC, UniRef100_UPI00075B5FE9, 
UniRef100_UPI00075B65B7, UniRef100_UPI00075B9178, UniRef100_UPI00075BE4DC, UniRef100_UPI00075C79AB, UniRef100_UPI00075CCDDB, UniRef100_UPI00075D0BE3, UniRef100_UPI00075D85B3, UniRef100_UPI00075DBA92, UniRef100_UPI00075FAA94, UniRef100_UPI000760FCE9, UniRef100_UPI0007618158, UniRef100_UPI00076A4F14, UniRef100_UPI00076AC068, UniRef100_UPI00076B80C6, UniRef100_UPI000773ACD7, UniRef100_UPI000778BF82, UniRef100_UPI00078203F9, UniRef100_UPI0007835402, UniRef100_UPI000784871F, UniRef100_UPI0007968E34, UniRef100_UPI00079774E6, UniRef100_UPI0007A4B40E, UniRef100_UPI0007A762BF, UniRef100_UPI0007AB27D7, UniRef100_UPI0007AC17F0, UniRef100_UPI0007AC296B, UniRef100_UPI0007BF1BDF, UniRef100_UPI0007BF909D, UniRef100_UPI0007C59919, UniRef100_UPI0007C874A2, UniRef100_UPI0007D84790, UniRef100_UPI0007D85A35, UniRef100_UPI0007E392F8, UniRef100_UPI0007E55B23, UniRef100_UPI0007E57A0D, UniRef100_UPI0007E934BB, UniRef100_UPI0007E94B5A, UniRef100_UPI0007E98D06, UniRef100_UPI0007EBB120, UniRef100_UPI0007ED48DE, UniRef100_UPI0007F94A3A, UniRef100_UPI000806113D, UniRef100_UPI000806426D, UniRef100_UPI0008073554, UniRef100_UPI00080ACA6E, UniRef100_UPI00080B1DA2, UniRef100_UPI00080FD493, UniRef100_UPI00080FE091, UniRef100_UPI0008100D1E, UniRef100_UPI000810121B, UniRef100_UPI000813515C, UniRef100_UPI0008141C3D, UniRef100_UPI0008243320, UniRef100_UPI000824925C, UniRef100_UPI0008258C9A, UniRef100_UPI0008261528, UniRef100_UPI000826EC5C, UniRef100_UPI000827126B, UniRef100_UPI00082A2C4A, UniRef100_UPI000837B000, UniRef100_UPI00083872DC, UniRef100_UPI0008391843, UniRef100_UPI00084BCB42, UniRef100_UPI00085292A3, UniRef100_UPI0008531F92, UniRef100_UPI000853D585, UniRef100_UPI00087894D8, UniRef100_UPI000878D386, UniRef100_UPI00088E874A, UniRef100_UPI000896D322, UniRef100_UPI000896FC68, UniRef100_UPI00089A4B27, UniRef100_UPI0008A25CF4, UniRef100_UPI0008A67938, UniRef100_UPI0008A7105B, UniRef100_UPI0008A95AAC, UniRef100_UPI0008AA05B0, UniRef100_UPI0008AA33F8, UniRef100_UPI0008AA40F7, UniRef100_UPI0008B1577D, UniRef100_UPI0008B4B3FB, UniRef100_UPI0008DC01EF, UniRef100_UPI0008DC25DB, UniRef100_UPI0008DF4110, UniRef100_UPI0008E0591E, UniRef100_UPI0008E167DF, UniRef100_UPI0008E3F465, UniRef100_UPI0008FD0FC3, UniRef100_UPI0008FD6178, UniRef100_UPI0008FE54A4, UniRef100_UPI0008FE98F2, UniRef100_UPI000903B1D7, UniRef100_UPI0009126DF1, UniRef100_UPI0009156948, UniRef100_UPI000925D0AE, UniRef100_UPI0009264FB5, UniRef100_UPI000927F4D4, UniRef100_UPI000929B92D, UniRef100_UPI00092BA78D, UniRef100_UPI00092BF398, UniRef100_UPI00093F1DB0, UniRef100_UPI00094620B5, UniRef100_UPI00094AD778, UniRef100_UPI00094ADEE6, UniRef100_UPI00096F648F, UniRef100_UPI000972CDBB, UniRef100_UPI00097C735D, UniRef100_UPI00097CBD84, UniRef100_UPI0009803707, UniRef100_UPI0009850070, UniRef100_UPI00098F5498, UniRef100_UPI00098F9F58, UniRef100_UPI0009918DDE, UniRef100_UPI0009918E80, UniRef100_UPI000991B843, UniRef100_UPI000991ECC0, UniRef100_UPI00099202E6, UniRef100_UPI0009925A62, UniRef100_UPI00099290EC, UniRef100_UPI000992C0C6, UniRef100_UPI000992EFC1, UniRef100_UPI0009933484, UniRef100_UPI0009938F51, UniRef100_UPI000993D9F8, UniRef100_UPI000993E357, UniRef100_UPI000998ACE2, UniRef100_UPI00099FF654, UniRef100_UPI0009A575BF, UniRef100_UPI0009A651B7, UniRef100_UPI0009A67221, UniRef100_UPI0009A71A35, UniRef100_UPI0009A7407F, 
UniRef100_UPI0009A76CBF, UniRef100_UPI0009A7BA19, UniRef100_UPI0009A7D2C6, UniRef100_UPI0009A81C1B, UniRef100_UPI0009A82F74, UniRef100_UPI0009A83D5C, UniRef100_UPI0009A84CAA, UniRef100_UPI0009A9889B, UniRef100_UPI0009A9E295, UniRef100_UPI0009AD4292, UniRef100_UPI0009B0EFA1, UniRef100_UPI0009B3E5F5, UniRef100_UPI0009B4862D, UniRef100_UPI0009B88550, UniRef100_UPI0009DDACEE, UniRef100_UPI0009E1EA7A, UniRef100_UPI0009E33B05, UniRef100_UPI0009EA339C, UniRef100_UPI0009F46E85, UniRef100_UPI0009F68776, UniRef100_UPI0009F9060E, UniRef100_UPI0009FB8949, UniRef100_UPI000A0183E7, UniRef100_UPI000A02F892, UniRef100_UPI000A03FD25, UniRef100_UPI000A060954, UniRef100_UPI000A110B1A, UniRef100_UPI000A1465EA, UniRef100_UPI000A21143E, UniRef100_UPI000A2F754F, UniRef100_UPI000A49ABD3, UniRef100_UPI000A5347FD, UniRef100_UPI000A5C7A3B, UniRef100_UPI000A6ED80F, UniRef100_UPI000A9D81C1, UniRef100_UPI000AAA1A51, UniRef100_UPI000AAE0E8D, UniRef100_UPI000AD95C66, UniRef100_UPI000AF050F7, UniRef100_UPI000AFB92EF, UniRef100_UPI000B10EA1D, UniRef100_UPI000B2010A0, UniRef100_UPI000B29CF81, UniRef100_UPI000B3FF204, UniRef100_UPI000B3FF40E, UniRef100_UPI000B4015A6, UniRef100_UPI000B404795, UniRef100_UPI000B404C7D, UniRef100_UPI000B404CF4, UniRef100_UPI000B48ACB2, UniRef100_UPI000B48EF3F, UniRef100_UPI000B4902CA, UniRef100_UPI000B490F16, UniRef100_UPI000B4B4EB0, UniRef100_UPI000B52E12F, UniRef100_UPI000B5A977E, UniRef100_UPI000B797195, UniRef100_UPI000B79E667, UniRef100_UPI000B7DE1A2, UniRef100_UPI000B8385AC, UniRef100_UPI000B83B41A, UniRef100_UPI000B862E7C, UniRef100_UPI000B897A65, UniRef100_UPI000B89D3FB, UniRef100_UPI000B978DEE, UniRef100_UPI000B97B73F, UniRef100_UPI000BA4FEC9, UniRef100_UPI000BAFEC6F, UniRef100_UPI000BB2EC15, UniRef100_UPI000BB36686, UniRef100_UPI000BBBE5FF, UniRef100_UPI000BBC4CA6, UniRef100_UPI000BBC8987, UniRef100_UPI000BBCB9FA, UniRef100_UPI000BBCC1E2, UniRef100_UPI000BC6149E, UniRef100_UPI000BE7BAAE, UniRef100_UPI000BE8F712, UniRef100_UPI000BEA4AB7, UniRef100_UPI000BFF0B5A, UniRef100_UPI000BFF6ED9, UniRef100_UPI000C239A0E, UniRef100_UPI000C25CF04, UniRef100_UPI000C25EEB8, UniRef100_UPI000C2606C3, UniRef100_UPI000C265010, UniRef100_UPI000C265436, UniRef100_UPI000C267AB9, UniRef100_UPI000C2693F5, UniRef100_UPI000C26B86E, UniRef100_UPI000C26BAC7, UniRef100_UPI000C290228, UniRef100_UPI000C31DE6A, UniRef100_UPI000C32CEF6, UniRef100_UPI000CD2F9A8, UniRef100_UPI000CD9CD2A, UniRef100_UPI000CDD1F00, UniRef100_UPI000CDEE421, UniRef100_UPI000CE98C81, UniRef100_UPI000CF89484, UniRef100_UPI000CF8BB9C, UniRef100_UPI000CF9D9D2, UniRef100_UPI000CFA7232, UniRef100_UPI000D0A9C15, UniRef100_UPI000D19941A, UniRef100_UPI000D1A214A, UniRef100_UPI000D1E7A18, UniRef100_UPI000D22095A, UniRef100_UPI000D22A437, UniRef100_UPI000D336121, UniRef100_UPI000D33B171, UniRef100_UPI000D343D84, UniRef100_UPI000D347C09, UniRef100_UPI000D34D906, UniRef100_UPI000D34F6EC, UniRef100_UPI000D35183D, UniRef100_UPI000D352CAF, UniRef100_UPI000D3597E3, UniRef100_UPI000D35F97D, UniRef100_UPI000D361772, UniRef100_UPI000D371BBF, UniRef100_UPI000D374299, UniRef100_UPI000D376519, UniRef100_UPI000D376F26, UniRef100_UPI000D383387, UniRef100_UPI000D38CBC8, UniRef100_UPI000D38D517, UniRef100_UPI000D38DA91, UniRef100_UPI000D391BA9, UniRef100_UPI000D399AB2, UniRef100_UPI000D39EDAF, UniRef100_UPI000D3A2F21, 
UniRef100_UPI000D3AF062, UniRef100_UPI000D3B42FB, UniRef100_UPI000D3B4617, UniRef100_UPI000D3BEDC1, UniRef100_UPI000D3BF81F, UniRef100_UPI000D3C5C7E, UniRef100_UPI000D3E42D3, UniRef100_UPI000D3EC359, UniRef100_UPI000D54EB1B, UniRef100_UPI000D6A0451, UniRef100_UPI000D6A8772, UniRef100_UPI000D6AA926, UniRef100_UPI000D6ABEBA, UniRef100_UPI000D6B50A6, UniRef100_UPI000D6C9481, UniRef100_UPI000D6CE158, UniRef100_UPI000D6D822C, UniRef100_UPI000D6F10DF, UniRef100_UPI000D71AD5D, UniRef100_UPI000D71C59C, UniRef100_UPI000D767CEA, UniRef100_UPI000D76958B, UniRef100_UPI000D7BA027, UniRef100_UPI000D7BC140, UniRef100_UPI000D7C02A9, UniRef100_UPI000D7C43DE, UniRef100_UPI000D7C60D2, UniRef100_UPI000D7C8D8D, UniRef100_UPI000D7CD63F, UniRef100_UPI000D7CD8D5, UniRef100_UPI000D7D08B3, UniRef100_UPI000D82A0F0, UniRef100_UPI000D82D63D, UniRef100_UPI000D83F2D0, UniRef100_UPI000D84D93C, UniRef100_UPI000D88D503, UniRef100_UPI000D88F2F1, UniRef100_UPI000D890A86, UniRef100_UPI000D9156D3, UniRef100_UPI000DA199AF, UniRef100_UPI000DA1C0AC, UniRef100_UPI000DA22DB4, UniRef100_UPI000DA252CF, UniRef100_W9AJK7, UniRef100_W9ATZ2, UniRef100_W9BKI5, UniRef100_W9CJZ3, UniRef100_W9CKY7, UniRef100_X5E5S5, UniRef100_X5KPW6, UniRef100_X5L6P7, UniRef100_X5L8J5, UniRef100_X5LFN9, UniRef100_X5LJ77, UniRef100_X5LNT2, UniRef100_X5M0W1 
Table S2 Sugar concentration in ryegrass root exudates.

\begin{tabular}{cccccccc}
\hline & Glucose & Fructose & Mannitol & Sucrose & Arabinose & Maltose & $\Sigma$ Sugar \\
\hline $\begin{array}{c}\text { Mean } \\
(\boldsymbol{\mu g} / \mathbf{g} \text { FW })\end{array}$ & 116.67 & 17.33333 & 8.67 & 133.00 & 7.33 & 5.00 & 288.00 \\
Std & 5.31 & 2.054805 & 2.05 & 7.79 & 0.47 & 1.63 & 14.17 \\
\hline
\end{tabular}


Table S3 Organic acid concentration in ryegrass root exudates.

\begin{tabular}{cccccccccc} 
& Oxalic & Lactic & Malic & Acetic & Succinic & Citric & Fumaric & Tartaric & $\sum$ Organic acid \\
\hline Mean ( $\mu$ g/g FW) & 559.67 & 2.20 & 30.33 & 16.67 & 3.33 & 0.33 & 27.33 & 58.33 & 698.20 \\
Std & 31.51 & 1.59 & 2.62 & 2.05 & 0.47 & 0.47 & 2.87 & 3.30 & 26.42 \\
\hline
\end{tabular}


Table S4 Taxonomic information of phenanthrene degraders.

\begin{tabular}{|c|c|}
\hline OTU ID & taxonomic information \\
\hline OTU113 & k_Bacteria; p_Proteobacteria; c_Alphaproteobacteria; o_Rhizobiales; f_Hyphomicrobiaceae; g_Rhodoplanes; s_ \\
\hline OTU173 & k_Bacteria; p_Proteobacteria; c_Alphaproteobacteria; o_Sphingomonadales; f_Sphingomonadaceae; g_Kaistobacter; s_ \\
\hline OTU191 & k_Bacteria; $\mathrm{p} \_$Acidobacteria; c_Acidobacteriia; o_Acidobacteriales; $\mathrm{f}$ _Acidobacteriaceae; $\mathrm{g}$; $\mathrm{s}$ _ \\
\hline OTU205 & k_Bacteria; p_Actinobacteria; c_Actinobacteria; o_Actinomycetales; f_Intrasporangiaceae; g_Terracoccus; s_ \\
\hline OTU218 & k_Bacteria; p_Proteobacteria; c_Alphaproteobacteria; o_Sphingomonadales; f_Sphingomonadaceae; g_Kaistobacter; s_ \\
\hline OTU236 & k_Bacteria; p_Acidobacteria; c_Acidobacteriia; o_Acidobacteriales; f_Acidobacteriaceae; g_; s_ \\
\hline OTU302 & k_Bacteria; p_Proteobacteria; c_Alphaproteobacteria; o_Rhizobiales; f_Phyllobacteriaceae; g_Mesorhizobium; s_ \\
\hline OTU32 & k_Bacteria; p_Acidobacteria; c_DA052; o_Ellin6513;f_g_; s_ \\
\hline OTU355 & k_Bacteria; p_Planctomycetes; c_Planctomycetia; o_Gemmatales; f_Isosphaeraceae; g_; s_ \\
\hline OTU38 & k_Bacteria; p_Proteobacteria; c_Betaproteobacteria; o_Burkholderiales; f_Oxalobacteraceae; g_Ralstonia; s_ \\
\hline OTU404 & k_Bacteria; p_Proteobacteria; c_Alphaproteobacteria; o_Sphingomonadales; f_Sphingomonadaceae \\
\hline OTU407 & k_Bacteria; p_Acidobacteria; c_Solibacteres; o_Solibacterales; f_Solibacteraceae; g_; s_ \\
\hline OTU428 & k_Bacteria; p_Actinobacteria; c_Thermoleophilia; o_Gaiellales; f_Gaiellaceae; g_; s_ \\
\hline OTU449 & k_Bacteria; p_Actinobacteria; c_Actinobacteria; o_Actinomycetales; f_Mycobacteriaceae; g_Mycobacterium; s_ \\
\hline OTU453 & k_Bacteria; p_Chloroflexi; c_Ktedonobacteria; o_JG30-KF-AS9; f_; g_; s_ \\
\hline OTU47 & k_Bacteria; p_Actinobacteria; c_Thermoleophilia; o_Solirubrobacterales; f_Conexibacteraceae \\
\hline OTU510 & k_Bacteria; p_Acidobacteria; c_Acidobacteriia; o_Acidobacteriales; f_Acidobacteriaceae; g_; s_ \\
\hline OTU526 & k_Bacteria; p_Chloroflexi; c_Ktedonobacteria; o_JG30-KF-AS9; f_;g_; s_ \\
\hline OTU54 & k_Bacteria; p_Actinobacteria; c_Actinobacteria; o_Actinomycetales; f_Pseudonocardiaceae; g_Pseudonocardia; s_ \\
\hline
\end{tabular}


Table S5 Relative abundance of all active phenanthrene degraders in NR and R treatments.

\begin{tabular}{cccc}
\hline Treatment & $\mathbf{3 ~ d}$ & $\mathbf{7 ~ d}$ & $\mathbf{1 4} \mathbf{d}$ \\
\hline $\mathbf{N R}$ & $0.15 \%$ & $2.1 \%$ & $7.9 \%$ \\
$\mathbf{R}$ & $1.3 \%$ & $1.3 \%$ & $1.8 \%$ \\
\hline
\end{tabular}


Table S6 The average $\mathrm{Ka} / \mathrm{Ks}$ ratios of phenanthrene degradation related genes and the $p$ values of one-way ANOVA.

\begin{tabular}{cccc}
\hline Gene group & Non-rhizosphere & Rhizosphere & $\boldsymbol{p}$ \\
\hline GROUP134 & 0.24 & 0.28 & $1.21 \times 10^{-8}$ \\
GROUP358 & 0.29 & 0.27 & $9.06 \times 10^{-4}$ \\
GROUP2045 & 0.19 & 0.10 & $8.72 \times 10^{-4}$ \\
GROUP2741 & 0.13 & 0.07 & $2.22 \times 10^{-2}$ \\
GROUP320 & 0.29 & 0.28 & $1.73 \times 10^{-2}$ \\
\hline
\end{tabular}


Table S7 The ten altered metabolic modules with top abundance and their corresponding $M$ value in Phenanthrene degraders and Total microbial community.

\begin{tabular}{|c|c|c|}
\hline Sample & $\begin{array}{c}\text { M } \\
\text { value }\end{array}$ & Module \\
\hline \multirow{10}{*}{ Phenanthrene degraders } & 1338.05 & M00009 Citrate cycle (TCA cycle, Krebs cycle) \\
\hline & 1113.08 & M00173 Reductive citrate cycle (Arnon-Buchanan cycle) \\
\hline & 1015.58 & M00011 Citrate cycle, second carbon oxidation, 2-oxoglutarate $=>$ oxaloacetate \\
\hline & 871.00 & M00048 Inosine monophosphate biosynthesis, PRPP + glutamine $=>$ IMP \\
\hline & 846.83 & M00165 Reductive pentose phosphate cycle (Calvin cycle) \\
\hline & 846.32 & M00001 Glycolysis (Embden-Meyerhof pathway), glucose $=>$ pyruvate \\
\hline & 815.95 & M00004 Pentose phosphate pathway (Pentose phosphate cycle) \\
\hline & 759.61 & M00121 Heme biosynthesis, plants and bacteria, glutamate $=>$ heme \\
\hline & 710.23 & M00083 Fatty acid biosynthesis, elongation \\
\hline & 701.72 & M00570 Isoleucine biosynthesis, threonine $=>$ 2-oxobutanoate $=>$ isoleucine \\
\hline \multirow{10}{*}{ Total microbial community } & 978.21 & M00009 Citrate cycle (TCA cycle, Krebs cycle) \\
\hline & 811.20 & M00173 Reductive citrate cycle (Arnon-Buchanan cycle) \\
\hline & 741.79 & M00011 Citrate cycle, second carbon oxidation, 2-oxoglutarate $=>$ oxaloacetate \\
\hline & 688.30 & M00001 Glycolysis (Embden-Meyerhof pathway), glucose $=>$ pyruvate \\
\hline & 661.75 & M00004 Pentose phosphate pathway (Pentose phosphate cycle) \\
\hline & 660.37 & M00048 Inosine monophosphate biosynthesis, PRPP + glutamine $=>$ IMP \\
\hline & 659.60 & M00165 Reductive pentose phosphate cycle (Calvin cycle) \\
\hline & 566.37 & M00026 Histidine biosynthesis, PRPP $=>$ histidine \\
\hline & 544.33 & M00083 Fatty acid biosynthesis, elongation \\
\hline & 541.25 & M00121 Heme biosynthesis, plants and bacteria, glutamate $\Rightarrow>$ heme \\
\hline
\end{tabular}




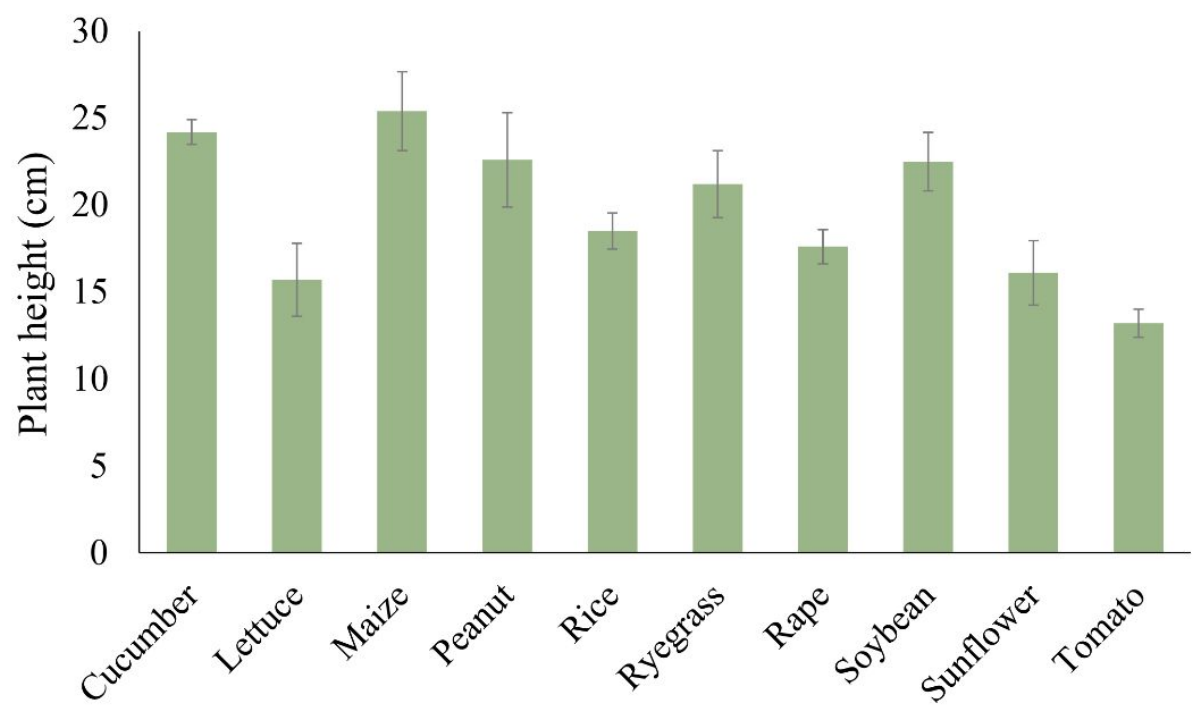

Figure S1 The heights of all the cultivated plants. 


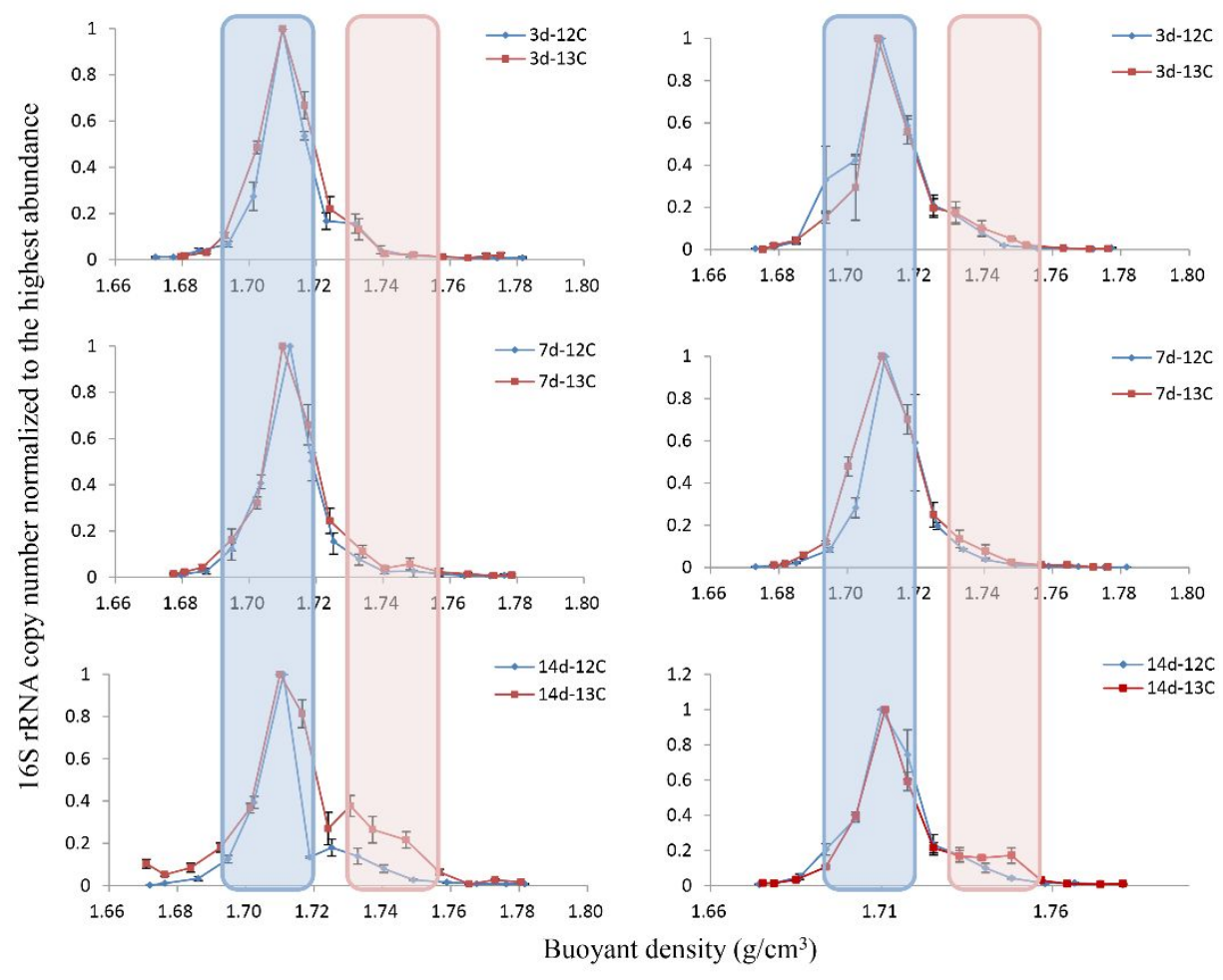

Figure S2 The copy number of 16S rRNA genes (normalized to the highest abundance) along with $\mathrm{CsCl}$ gradient fractions in $\mathrm{NR}$ and $\mathrm{R}$ treatments. 


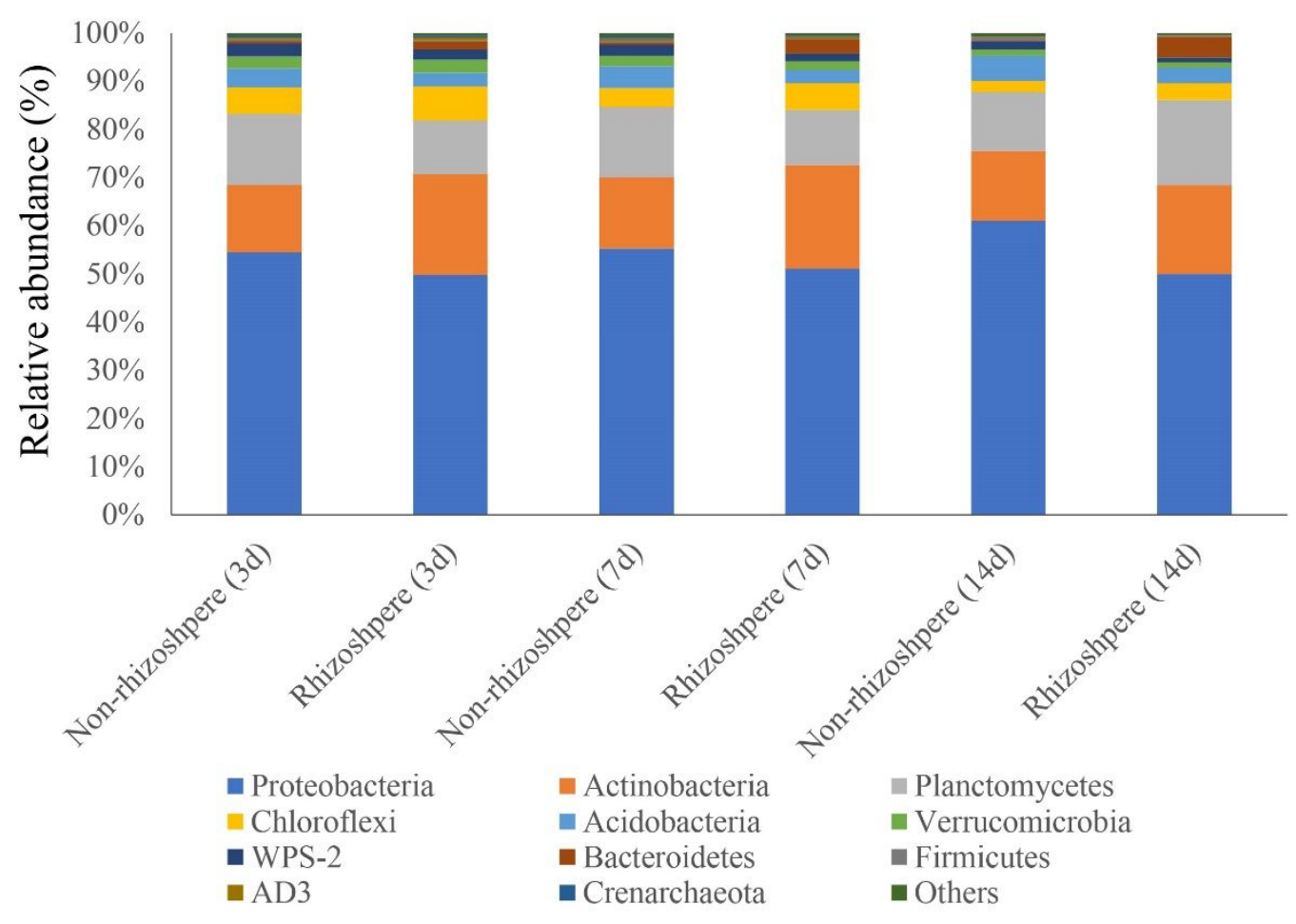

Figure S3 Dominant microbes $(>1 \%$ o) on phyla level in R and NR treatments. 


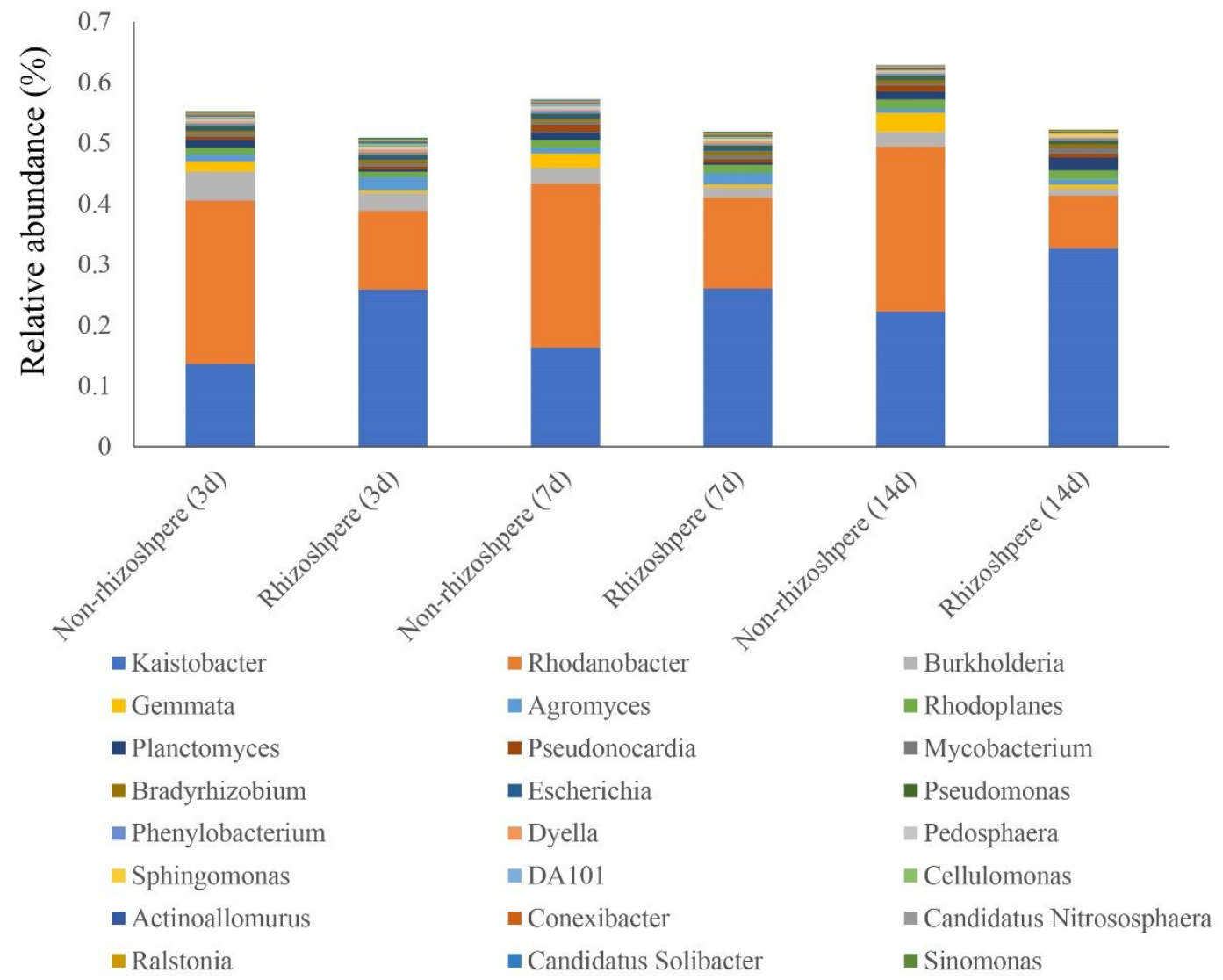

Figure S4 Dominant microbes $(>1 \%$ ) on genus level in R and NR treatments. 


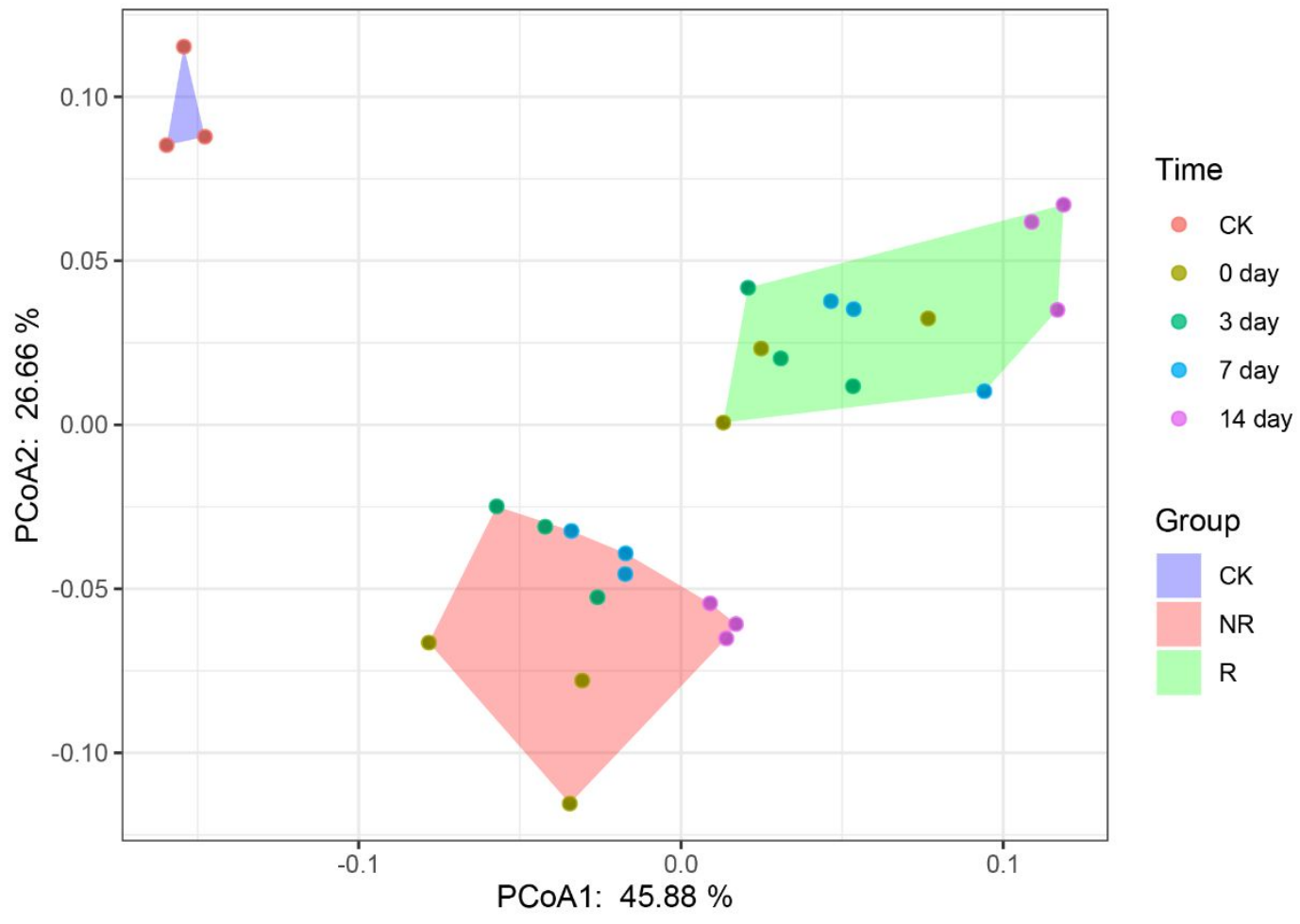

Figure S5 Score plot of PCoA for microbial communities from original soil, NR and $\mathrm{R}$ treatments. 


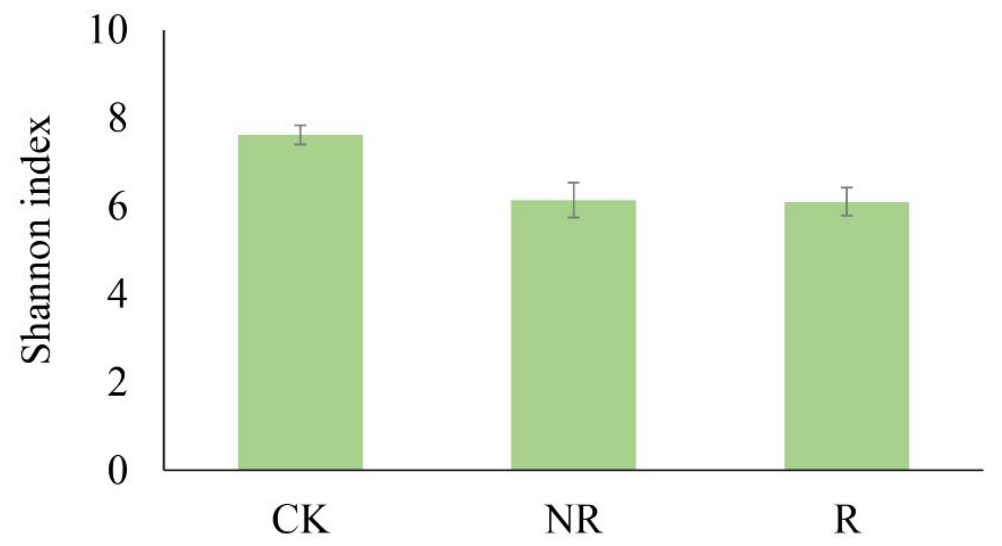

Figure S6 Shannon index in original soil, NR and R treatments. 


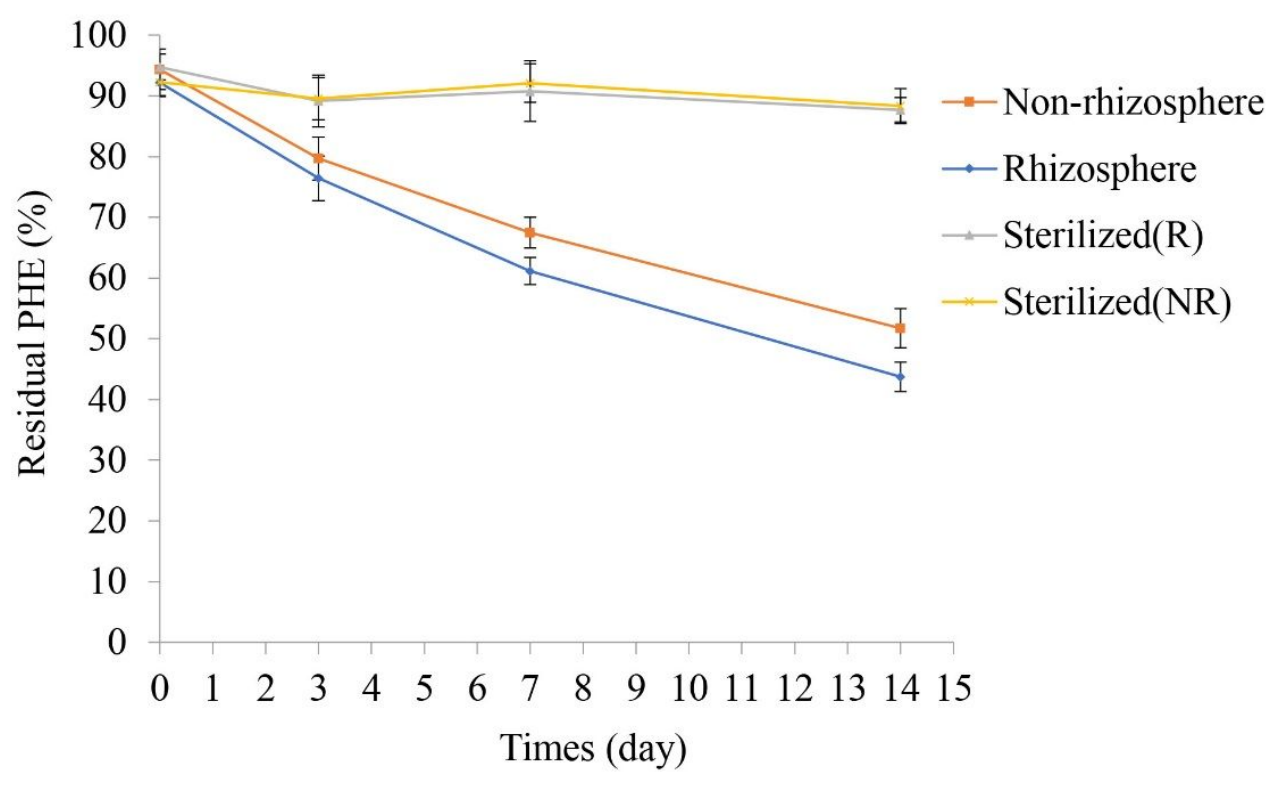

Figure S7 Residual phenanthrene percentage in R, NR and sterilized samples. 
A

Tree scale: 0.01

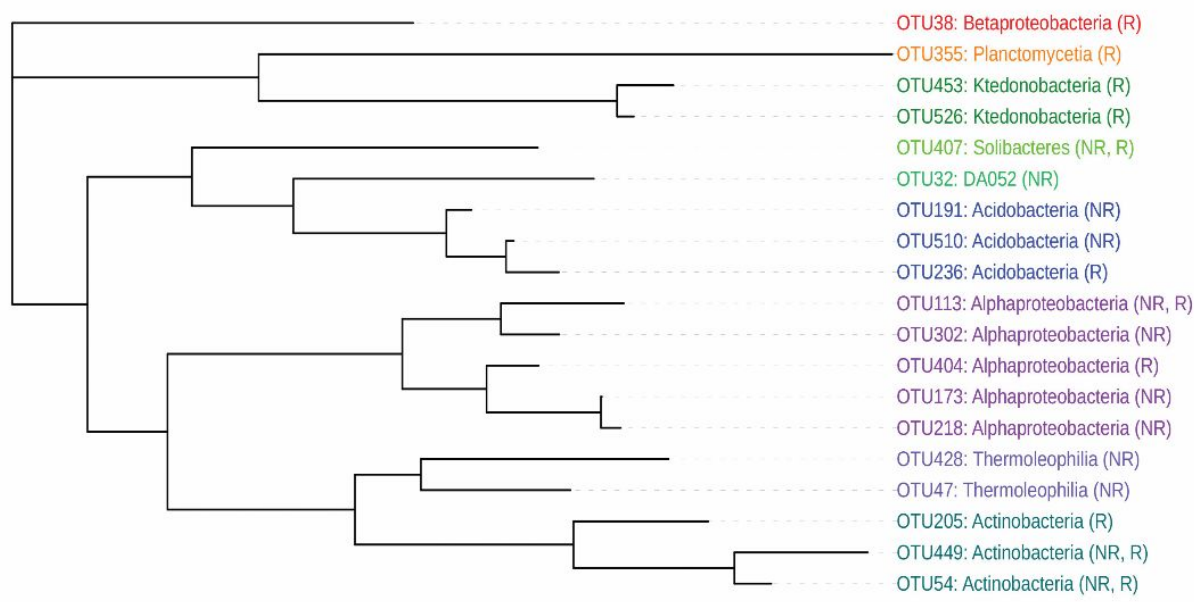

B

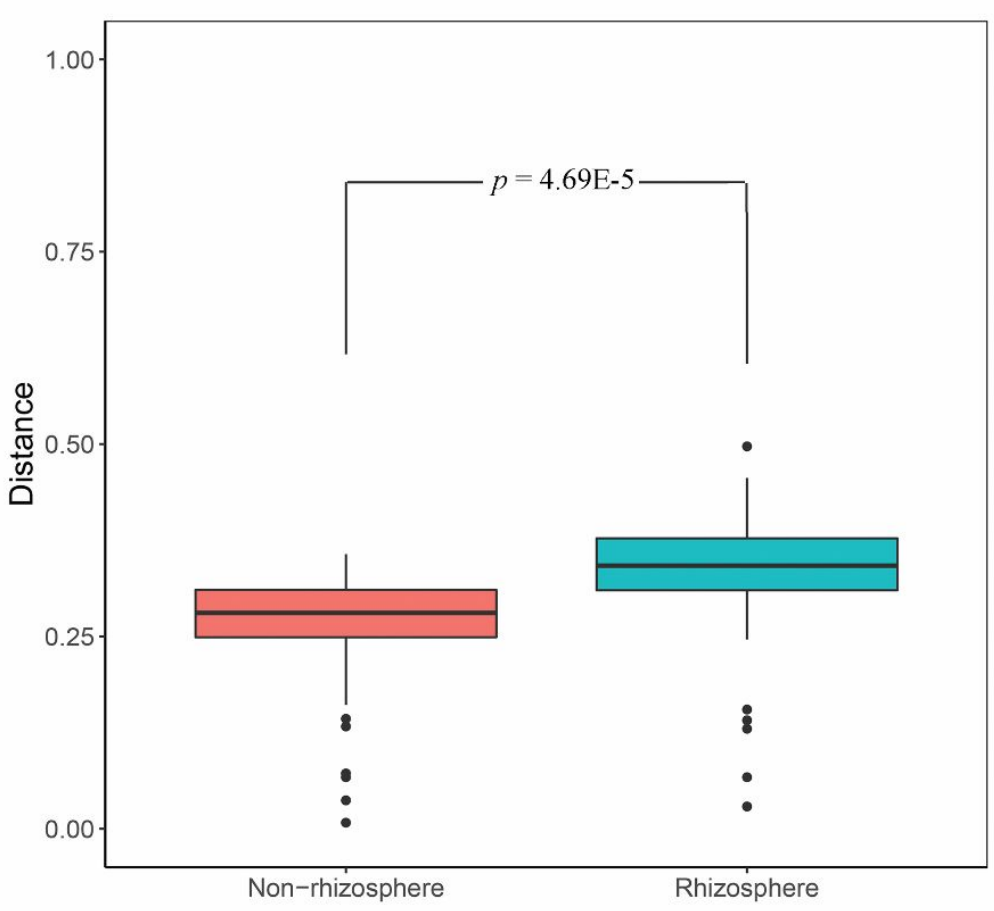

Figure S8 A) Phylogenetic tree of identified OTUs responsible for in situ phenanthrene degradation in NR and $\mathrm{R}$ treatments. B) Differences in phylogenetic distance between NR and R treatments. 


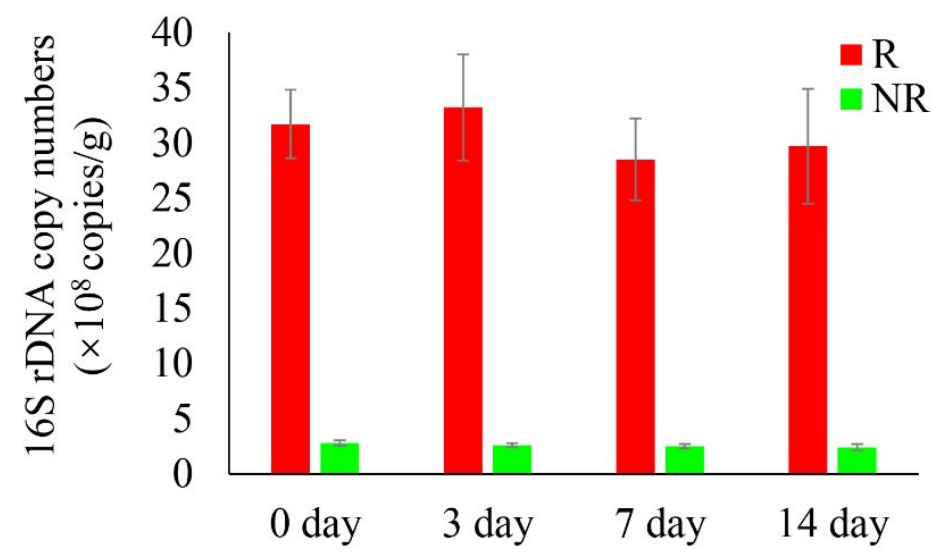

Figure S9 The copy numbers of $16 \mathrm{~S}$ rRNA genes on day $0,3,7$ and 14 between $\mathrm{R}$ and NR treatments. 


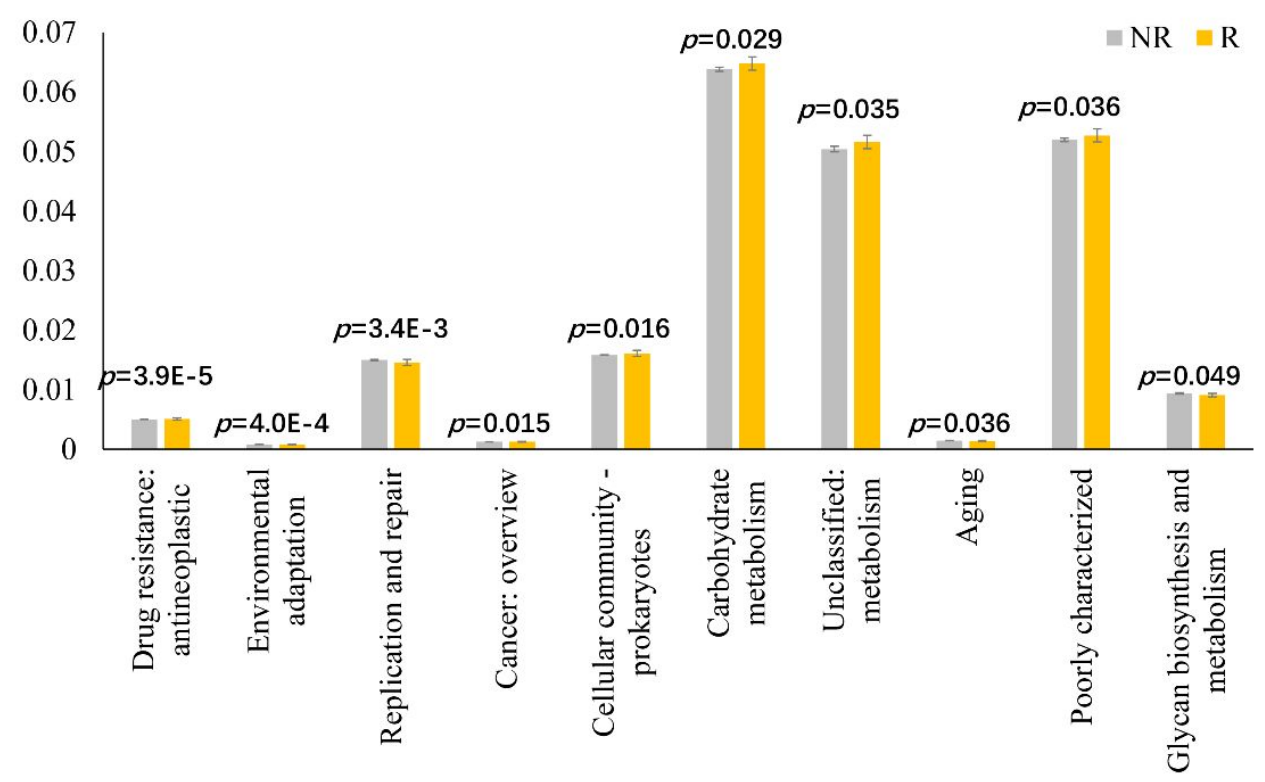

Figure S10 The 10 different abundance modules between NR and R treatments. 


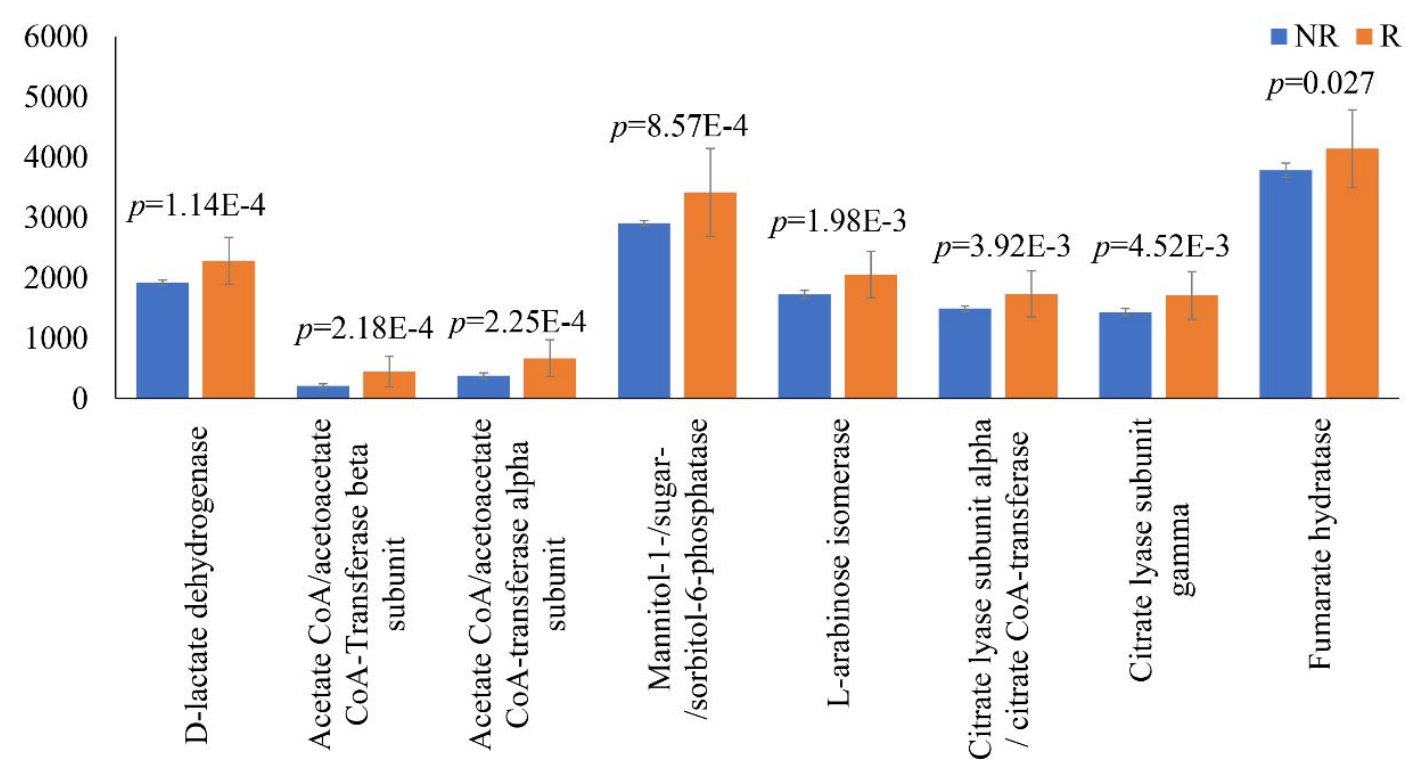

Figure S11 Root exudates metabolism related genes enriched in R treatment. 